\title{
RECURRENCE OF A WEIGHTED RANDOM WALK ON A CIRCLE PACKING WITH PARABOLIC CARRIER
}

\author{
ORI GUREL-GUREVICH AND MATAN SEIDEL
}

\begin{abstract}
In this paper we show that given a circle packing of an infinite planar triangulation such that its carrier is parabolic, placing weights on the edges according to a certain natural way introduced by Dubejko, makes the random walk recurrent. We also propose a higher-dimensional analogue of the Dubejko weights.
\end{abstract}

\section{INTRODUCTION}

A circle packing is a collection of circles in the plane with disjoint interiors. The tangency graph of a circle packing is the graph obtained by assigning a vertex to each circle and connecting two vertices by an edge if their respective circles are tangent to one another. A planar graph is called a triangulation if it admits a drawing in the plane (also called a triangulation) in which all faces are incident to exactly 3 edges, outer face included. The celebrated circle packing theorem [11, 15] asserts that every finite planar graph is the tangency graph of some circle packing. Furthermore, if the graph is a triangulation then its circle packing is unique up to Möbius transformations and reflections. A concise background on the probabilistic and combinatorial properties of circle packings can be found in [13].

Infinite planar graphs can also be shown (see [13]) to be isomorphic to the tangency graph of some (infinite) circle packing. However, the question of uniqueness becomes more complicated, and requires a few more definitions. All infinite graphs in this paper are assumed to be connected and locally finite, and infinite triangulations are assumed to have no outer face. For an infinite triangulation drawn in the plane we use the term face to also mean the compact set bounded by its edges. A circle packing induces a drawing in straight lines of its tangency graph by mapping the vertices to the centers of their corresponding circles. If this drawing is an infinite triangulation and the union of its faces is a domain $\Omega \subseteq \mathbb{R}^{2}$, the circle packing is said to be a circle-packed infinite triangulation of $\Omega, \Omega$ is called its carrier and the tangency graph is said to be circle-packable in $\Omega$.

In [8] and [9], He \& Schramm extended the circle packing theorem to infinite triangulations that are one-ended, i.e. such that the removal of any finite set of vertices leaves the graph with exactly one infinite connected component (and possibly more finite ones). They showed that the possible carriers of a circle packing of such a triangulation are deeply linked to properties of the simple random walk on the graph. A graph is said to be recurrent if the simple random walk started at some vertex $\rho$ almost surely returns to $\rho$ infinitely often, and transient otherwise. Indeed, among their results, they showed that for a bounded-degree one-ended triangulation, either the graph is recurrent and is circle-packable in the plane, or it is transient and circle-packable in the open unit disk. Another of their results shows that in the transient case, the graph is also circle-packable in the open unit square (or any other simply-connected domain strictly contained in the plane). Consequently, since Möbius transformations and reflections cannot map the unit disk to the unit square, one cannot hope for the same rigidity as in the finite case.

When removing the assumption of one-endedness, some more definitions are needed: A domain $\Omega \subseteq \mathbb{R}^{2}$ is called parabolic if for any open set $U \subseteq \Omega$, Brownian motion started at any point of $\Omega$ and killed at $\partial \Omega$ hits $U$ almost surely. The domain is said to be hyperbolic otherwise. An equivalent formulation for parabolicity is given in Proposition 5.13. In [7], Gurel-Gurevich, Nachmias \& Souto showed that a dichotomy still holds without oneendedness for infinite triangulations of bounded degree: the graph is recurrent iff the carrier of any circle packing of it is parabolic. Their proof relied on the Rodin-Sullivan [14] Ring Lemma, which shows that when the degree 
is bounded, the radii of adjacent circles must be comparable in length. Indeed, removing the bounded degree assumption may cause the theorem to fail. For example, as described in [13], we can add circles to the circle packing of the (recurrent) hexagonal lattice in a way that creates a drift in the random walk in a direction of choice, rendering it transient. However, the carrier has remained parabolic, being the entire plane.

Definition 1.1. A network is a pair $(G, c)$ where $G=(V, E)$ is a connected graph (with self-loops allowed) and $c: E \rightarrow(0, \infty)$ is a weight function on the edges. The weighted random walk on a network is the Markov chain with state space $V$ and transition probabilities $P_{x, y}=\frac{c_{x y}}{\pi(x)}$ where $\pi(x)=\sum_{y \sim x} c_{x y}$. The network is said to be recurrent if the weighted random walk started at some vertex $\rho \in V$ almost surely returns to $\rho$ infinitely often, and transient otherwise.

In [3], Dubejko proposed a way to place weights on the edges of a circle-packed infinite triangulation such that in the weighted random walk on the network, the sequence of centers of the circles visited becomes a martingale. These weights also arise naturally in the context of discrete complex analysis (see [4]). For completeness, we provide Dubejko's elegant proof here as Theorem 2.4. Let us precisely define the weights. In the straight-line drawing induced by a circle-packed infinite triangulation, each face $f$ is a straight-edge triangle. Thus, a circle packing for the dual graph is induced by mapping each face $f$ to its incircle. In the drawings of the graph and its dual, an edge $e$ and its dual $e^{\dagger}$ are orthogonal straight lines, as shown in Figure 1.1:

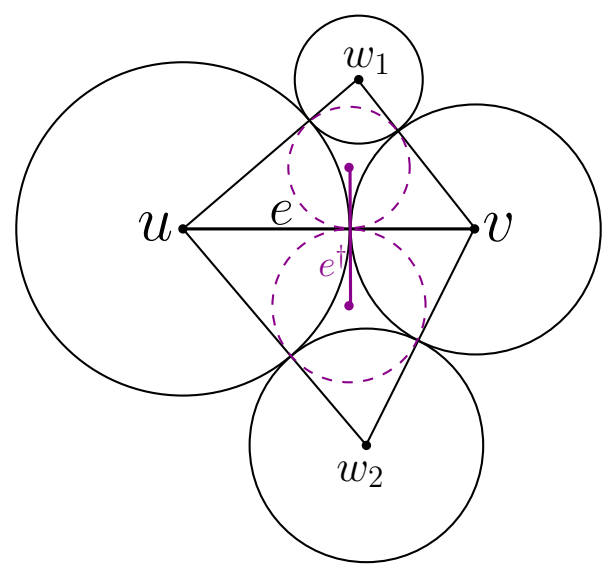

Figure 1.1. Part of a circle-packed triangulation in black and the induced circle packing of the dual graph in purple. The dual edge $e^{\dagger}$ of $e=u v$ connects the incenters of the two faces incident to $e$.

Definition 1.2. Let $\left\{C_{v}\right\}_{v \in V}$ be a circle-packed infinite triangulation. The Dubejko weight of an edge $e \in E$ is $c_{e}=\frac{\left|e^{\dagger}\right|}{|e|}$, where $|e|$ and $\left|e^{\dagger}\right|$ are the respective lengths of the straight line segments that $e$ and its dual edge $e^{\dagger}$ are mapped to.

Proposition 2.8 shows that the weights are bounded from above by a constant. Thus, Rayleigh monotonicity implies that if the simple random walk on a circle-packed triangulation is recurrent then so is the weighted random walk (WRW). Furthermore, as shown in Proposition 2.10, when the graph has bounded degree the weights are also bounded from below by a constant, and so in the bounded degree case the SRW and WRW are either both recurrent or both transient. So one might hope that replacing the SRW with the WRW would make the theorem of Gurel-Gurevich, Nachmias \& Souto hold for both the bounded degree and unbounded degree cases. The main goal of this paper is to prove the following:

Theorem 1.3. Let $\Omega \subseteq \mathbb{R}^{2}$ be a parabolic domain. Then for any circle-packed infinite triangulation of $\Omega$, the Dubejko-weighted random walk is recurrent. 
In section 2 we analyze some geometric properties of the weights. In section 3 we prove two special cases: that if the carrier is the entire plane then the WRW is recurrent, and that if the carrier is bounded then the WRW is transient. In section 4 we prove some lemmas on integration of harmonic functions needed for the proof of Theorem 1.3, which is proven itself in section 5. Finally, in section 6, we propose an analogue of the Dubejko weights for higher dimensions, and prove they make the weighted random walk into a martingale.

\section{Some Geometric Lemmas}

Remark 2.1. In the context of a circle packing, we use interchangeably the vertices of the tangency graph and the centers of their corresponding circles in $\mathbb{R}^{2}$.

Definition 2.2. Let $\left\{C_{v}\right\}_{v \in V}$ be a circle-packing of an infinite triangulation. For a vertex $v \in V$, the polygon of $v$ denoted by $P_{v}$ is the polygon whose sides are the dual edges to all the edges incident to $v$.

Remark 2.3. $P_{v}$ is convex, its vertices are the incenters of the faces incident to $v$ and the circle $C_{v}$ is inscribed in it.

We now provide our main probabilistic motivation for looking at the Dubejko weights, taken from [3]. An analogue for higher dimensions of this theorem is proven in Proposition 6.4.

Theorem 2.4. Let $\left\{C_{v}\right\}_{v \in V}$ be a circle-packed infinite triangulation. Let $\left(Z_{n}\right)_{n \in \mathbb{N}}$ be the sequence of vertices visited during a Dubejko-weighted random walk on $\left\{C_{v}\right\}_{v \in V}$. Then $\left(Z_{n}\right)_{n \in \mathbb{N}}$ is a martingale.

Proof. Set $\pi(x)=\sum_{y \sim x} c_{x y}$ and denote the transition probabilities by $p_{x y}=\frac{c_{x y}}{\pi(x)}$. Let $v \in V$, and let $u_{1}, u_{2}, \ldots, u_{n} \in$ $V$ be its neighbors in $G$. For each $i \in\{1,2, \ldots, n\}$ let $\overrightarrow{e_{i}}=u_{i}-v$ be the edge $v u_{i}$ oriented from $v$ to $u_{i}$. Let $R: \mathbb{R}^{2} \rightarrow \mathbb{R}^{2}$ be the linear clockwise rotation by $\frac{\pi}{2}$ radians, and let $\overrightarrow{f_{i}}$ be the dual edge of $\overrightarrow{e_{i}}$ oriented in a direction such that $R\left(\widehat{e}_{i}\right)=\widehat{f}_{i}$, where $\widehat{e_{i}}$ and $\widehat{f}_{i}$ are the respective unit vectors of $\vec{e}_{i}$ and $\vec{f}_{i}$.

It is enough to show that $\sum_{i=1}^{n} p_{v u_{i}} \overrightarrow{e_{i}}=0$. We have:

$$
R\left(\sum_{i=1}^{n} p_{v u_{i}} \overrightarrow{e_{i}}\right)=R\left(\frac{1}{\pi(v)} \sum_{i=1}^{n} \frac{\left\|\overrightarrow{f_{i}}\right\|}{\left\|\overrightarrow{e_{i}}\right\|} \cdot \overrightarrow{e_{i}}\right)=\frac{1}{\pi(v)} \sum_{i=1}^{n}\left\|\overrightarrow{f_{i}}\right\| R\left(\widehat{e_{i}}\right)=\frac{1}{\pi(v)} \sum_{i=1}^{n} \overrightarrow{f_{i}} .
$$

Since $\overrightarrow{f_{1}}, \overrightarrow{f_{2}}, \ldots, \overrightarrow{f_{n}}$ trace a closed curve along the boundary of the polygon $P_{v}$, their sum vanishes. Since $R$ is injective, the Theorem follows.

We continue by analyzing some of the properties of the Dubejko weights. Since the weights are defined using incircles, we will need the following formula for their radius:

Proposition 2.5. Let $x, y, z \in \mathbb{R}^{2}$ be centers of mutually tangent circles with respective radii $r_{x}, r_{y}, r_{z}$. Then the inradius of the triangle $x y z$ is:

$$
r=\sqrt{\frac{r_{x} r_{y} r_{z}}{r_{x}+r_{y}+r_{z}}}
$$

Proof. We calculate the the area $A$ of the triangle $x y z$ in two ways. On the one hand, the line segments connecting the incenter to $x, y, z$ divide the triangle into 3 triangles with altitudes $r$, and so:

$$
A=r \cdot\left(r_{x}+r_{y}+r_{z}\right) .
$$

On the other hand, by Heron's formula we know:

$$
A=\sqrt{\left(r_{x}+r_{y}+r_{z}\right) r_{x} r_{y} r_{z}} .
$$

Equating the two above and solving for $r$ finishes the proof.

The weights of the Dubejko-weighted random walk can be directly expressed using the radii by the following formula, appearing in [15] after Theorem 18.3: 
Proposition 2.6. Let $\left\{C_{v}\right\}_{v \in V}$ be a circle-packed infinite triangulation, and let $e=u v \in E$. Let $w_{1}, w_{2} \in V$ be the two vertices such that for every $i \in\{1,2\}, w_{i}$ forms a face with $u$ and $v$. Then the weight $c_{e}$ is:

$$
c_{e}=\frac{\sqrt{r_{u} r_{v}}}{r_{u}+r_{v}} \cdot\left(\sqrt{\frac{R_{1}}{r_{u}+r_{v}+R_{1}}}+\sqrt{\frac{R_{2}}{r_{u}+r_{v}+R_{2}}}\right),
$$

where $r_{u}, r_{v}, R_{1}, R_{2}$ are the radii of $C_{u}, C_{v}, C_{w_{1}}, C_{w_{2}}$ respectively.

Proof. For $i \in\{1,2\}$ set $r_{u v w_{i}}$ to be the inradius of the triangle $u v w_{i}$. Using Proposition 2.5, we can write:

$$
c_{e}=\frac{\left|e^{\dagger}\right|}{|e|}=\frac{r_{u v w_{1}}+r_{u v w_{2}}}{r_{u}+r_{v}}=\frac{\sqrt{r_{u} r_{v}}}{r_{u}+r_{v}} \cdot\left(\sqrt{\frac{R_{1}}{r_{u}+r_{v}+R_{1}}}+\sqrt{\frac{R_{2}}{r_{u}+r_{v}+R_{2}}}\right) .
$$

Lemma 2.7. Let $x, y, z \in \mathbb{R}^{2}$ be centers of mutually tangent circles with radii $r_{x}, r_{y}, r_{z}$, and let $r$ be the inradius of the triangle xyz. Then:

$$
r<\min \left\{\sqrt{r_{x} r_{y}}, \sqrt{r_{x} r_{z}}, \sqrt{r_{y} r_{z}}\right\} .
$$

Proof. By Proposition 2.5, we can write:

$$
r=\sqrt{r_{x} r_{y}} \cdot \sqrt{\frac{r_{z}}{r_{x}+r_{y}+r_{z}}}<\sqrt{r_{x} r_{y}} .
$$

A similar calculation replacing the role of $z$ with $x, y$ shows $r<\sqrt{r_{y} r_{z}}, r<\sqrt{r_{x} r_{z}}$, finishing the proof.

Proposition 2.8. Let $\left\{C_{v}\right\}_{v \in V}$ be a circle-packed infinite triangulation, and $G=(V, E)$ its tangency graph. Then for every edge $e \in E$ the weight is bounded from above by:

$$
c_{e}<1
$$

Proof. Write $e=u v$ for $u, v \in V$, and let $w_{1}, w_{2} \in V$ be the two vertices forming a face with $u, v$, as in Figure 1.1. Set $r_{u}, r_{v}$ to be the radii of $C_{u}, C_{v}$ and for each $i \in\{1,2\}$ set $r_{u v w_{i}}$ to be the inradius of the triangle $u v w_{i}$. The edge $e$ divides the dual edge $e^{\dagger}$ into two line segments of lengths $r_{u v w_{1}}$ and $r_{u v w_{2}}$. Using Lemma 2.7 and the AM-GM inequality we bound:

$$
c_{e}=\frac{\left|e^{\dagger}\right|}{|e|}=\frac{r_{u v w_{1}}+r_{u v w_{2}}}{r_{u}+r_{v}}<\frac{2 \sqrt{r_{u} r_{v}}}{r_{u}+r_{v}} \leq \frac{r_{u}+r_{v}}{r_{u}+r_{v}}=1 .
$$

A key result in the theory of circle packings is the famous Ring Lemma, proven by Rodin and Sullivan in [14]:

Lemma 2.9. (Ring Lemma): For each $d \in \mathbb{N}$, there exists some $r=r(d)>0$ such that if a unit circle is surrounded by $d$ circles forming a cycle externally tangent to it, as in Figure 2.1, then the radius of each of the $d$ circles is larger than $r$.

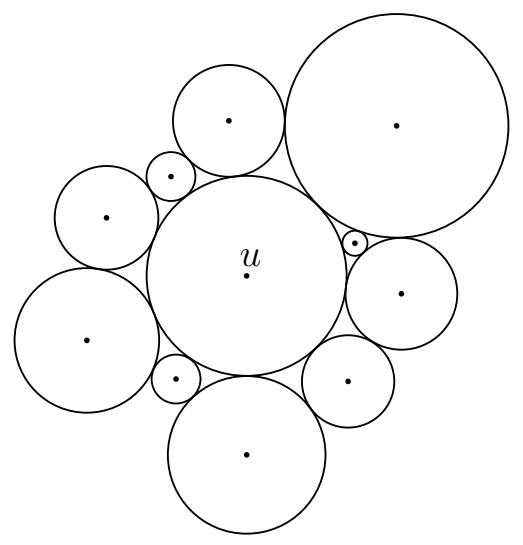

FiguRE 2.1. Circle $u$ surrounded by $d=10$ circles externally tangent to it 
The following consequence of the Ring Lemma shows that under the bounded degree assumption, since the radii of the circles around a given circle cannot be too small, the corresponding edge weights cannot be too small either:

Proposition 2.10. Let $\left\{C_{v}\right\}_{v \in V}$ be a circle-packed infinite triangulation and $G=(V, E)$ its tangency graph. If $G$ has degree bounded by $d$, then the weights are bounded from below by some constant $c_{0}=c_{0}(d)>0$.

Proof. Under the conditions of the Proposition, each circle in the packing is surrounded by a cycle of length at most $d$ of externally tangent circles. Thus, for each pair of adjacent circles, we can apply the Ring Lemma 2.9 in the wanted direction and deduce that the ratio of their respective radii is smaller than some global $M=M(d)>0$ (which can be taken to be the inverse of $r(d)$ for example).

Let $e=u v \in E$ be an edge and let $w_{1}, w_{2}$ the two vertices such that $u v w_{1}$ and $u v w_{2}$ are faces of the circle packing's drawing. Then using the formula from Proposition 2.6 for the weights we bound:

$$
\begin{aligned}
c_{e} & =\sqrt{\frac{r_{u}}{r_{u}+r_{v}}} \cdot \sqrt{\frac{r_{v}}{r_{u}+r_{v}}} \cdot\left(\sqrt{\frac{R_{1}}{r_{u}+r_{v}+R_{1}}}+\sqrt{\frac{R_{2}}{r_{u}+r_{v}+R_{2}}}\right) \geq \\
& \geq \sqrt{\frac{r_{u}}{r_{u}+M r_{u}}} \cdot \sqrt{\frac{r_{v}}{M r_{v}+r_{v}}} \cdot\left(\sqrt{\frac{R_{1}}{M R_{1}+M R_{1}+R_{1}}}+\sqrt{\frac{R_{2}}{M R_{2}+M R_{2}+R_{2}}}\right)= \\
& =\frac{2}{M+1} \sqrt{\frac{1}{2 M+1}},
\end{aligned}
$$

so we can take $c_{0}=\frac{2}{M+1} \sqrt{\frac{1}{2 M+1}}$.

Proposition 2.11. Let $\left\{C_{v}\right\}_{v \in V}$ be a circle-packed infinite triangulation and $G=(V, E)$ its tangency graph, and let $\left\{c_{e}\right\}_{e \in E}$ be the Dubejko weights induced by the circle packing. Then for any vertex $v \in V$ the sum of the weights around $v$ is bounded by a constant:

$$
\sum_{u \sim v} c_{v u}<2 \pi
$$

Proof. The weights are invariant under dilation, so we can assume without loss of generality that the radius of $C_{v}$ is 1 . Let $u_{1}, u_{2} \in V$ be two vertices sharing a face with $v$, as drawn in Figure 2.2:

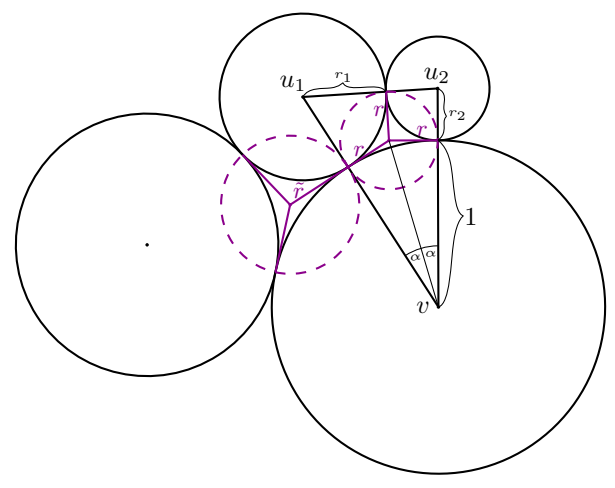

FIGURE 2.2. Triangle $v u_{1} u_{2}$ and its incircle

Denote by $r_{1}, r_{2}$ their respective radii. Let $r$ be the inradius of the triangle $v u_{1} u_{2}$. Then by Proposition 2.5 we have:

$$
r=\sqrt{\frac{r_{1} r_{2}}{1+r_{1}+r_{2}}}
$$

Denote by $2 \alpha$ the angle $\angle u_{1} v u_{2}$. The incenter is the point of concurrency of the angle bisectors, so we have a right-angled triangle with legs of length 1 and $r$ and angle $\alpha$, so $r=\tan \alpha$. Combining the two formulae for $r$ we get:

$$
\frac{1}{\cos ^{2} \alpha}=1+\tan ^{2} \alpha=1+r^{2}=\frac{1+r_{1}+r_{2}+r_{1} r_{2}}{1+r_{1}+r_{2}}=\frac{\left(1+r_{1}\right)\left(1+r_{2}\right)}{1+r_{1}+r_{2}}
$$


We think of the weight $c_{v u_{1}}$ as "split" between two edges connected in parallel with weights $\frac{r}{1+r_{1}}$ and $\frac{\tilde{r}}{1+r_{1}}$ where $\tilde{r}$ is the inradius of the triangle from the other side of the edge $v u_{1}$. We can now bound the sum of the conductances of the two half-edges relevant to the triangle $v u_{1} u_{2}$ :

$$
\frac{r}{1+r_{1}}+\frac{r}{1+r_{2}}=r \cdot \frac{2+r_{1}+r_{2}}{\left(1+r_{1}\right)\left(1+r_{2}\right)}<r \cdot \frac{2+2 r_{1}+2 r_{2}}{\left(1+r_{1}\right)\left(1+r_{2}\right)}=\tan \alpha \cdot 2 \cos ^{2} \alpha=\sin (2 \alpha)<2 \alpha .
$$

Summing the last inequality over all faces incident to $v$ gives the desired result.

Remark 2.12. The inequality of Proposition 2.11 is in fact tight: Consider a unit circle surrounded by $n$ identical circles of radius $r_{n}$. Denote by $P_{n}$ the perimeter of an $n$-regular polygon with inscribed radius 1 , then since $\lim _{n \rightarrow \infty} r_{n}=0$ and $\lim _{n \rightarrow \infty} P_{n}=2 \pi$, the sum of weights around the unit circle is:

$$
\frac{1}{1+r_{n}} \cdot P_{n} \underset{n \rightarrow \infty}{\longrightarrow} 2 \pi
$$

Lemma 2.13. Let $x, y, z \in \mathbb{R}^{2}$ be centers of mutually tangent circles with radii $r_{x}, r_{y}, r_{z}$, and let $r, M$ be the inradius and incenter of the triangle $x y z$. Denote by $Q, R$ the respective tangency points of the incircle with the sides $x y, x z$. Then:

$$
\measuredangle Q M R>2 \tan ^{-1}\left(\sqrt{\frac{r_{x}}{\min \left\{r_{y}, r_{z}\right\}}}\right) .
$$

In the context of a circle packing, this Lemma tells us that for a given face $x y z$, if at least one of $y, z$ has a comparable radius to $x$ then the angle of the polygon $P_{x}$ at the incenter of $x y z$ cannot be too small.

Proof. Set $\alpha=\measuredangle Q M R$ and consider the right triangle $x M Q$ : The lengths of its altitudes are $|M Q|=r$ and $|x Q|=r_{x}$. The line $x M$ bisects the angle $\measuredangle Q M R$ and so:

$$
\frac{r_{x}}{r}=\tan \measuredangle Q M x=\tan \frac{\alpha}{2} .
$$

Using Lemma 2.7 we bound:

$$
\tan \frac{\alpha}{2}>\frac{r_{x}}{\sqrt{r_{x} r_{y}}}=\sqrt{\frac{r_{x}}{r_{y}}}
$$

and since $\tan ^{-1}$ is strictly increasing we get $\alpha>2 \tan ^{-1}\left(\sqrt{\frac{r_{x}}{r_{y}}}\right)$. A similar calculation replacing $y$ with $z$ shows $\alpha>2 \tan ^{-1}\left(\sqrt{\frac{r_{x}}{r_{z}}}\right)$, and the result follows.

The following beautiful theorem by Descartes (see $§ 1.5$ in [2]), illustrated in Figure 2.3, will also be of use to us:

Theorem 2.14. (Descartes' Theorem): Let $k_{1}, k_{2}, k_{3}$ be the curvatures (i.e. the reciprocal of the radius) of three circles in the plane externally tangent to one another in 3 distinct points. Then there are exactly two other circles (or one circle and one line) tangent to all three, and their curvatures $k$ satisfy:

$$
k=k_{1}+k_{2}+k_{3} \pm 2 \sqrt{k_{1} k_{2}+k_{2} k_{3}+k_{3} k_{1}}
$$

where positive $k$, negative $k$ and $k=0$ represent a circle tangent externally to all three, internally to all three and a straight line respectively. 


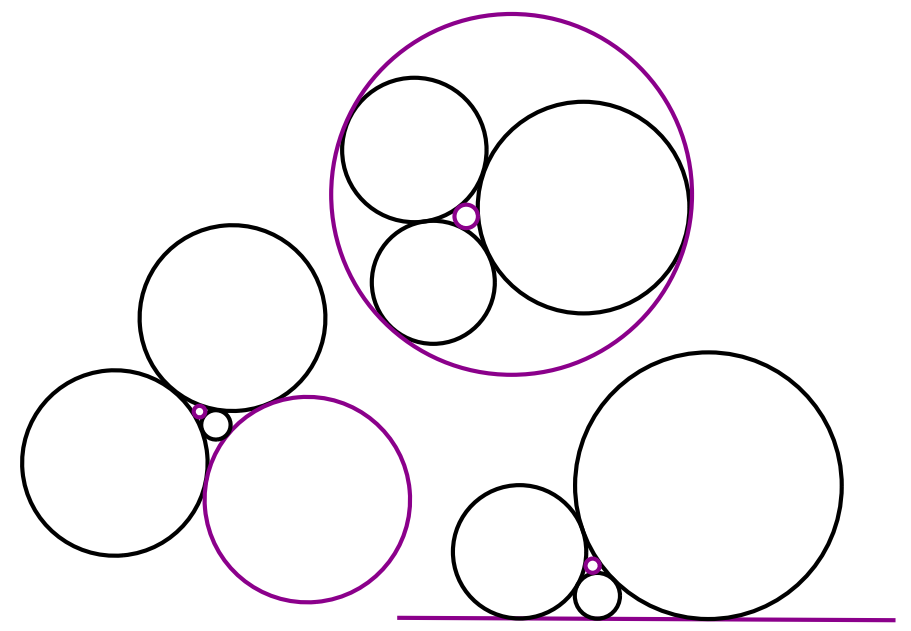

FIGURE 2.3. 3 mutually-tangent circles in black and the 2 possibilities for a 4 th circle in purple. A negative-curvature solution can be seen in the topmost example and a zero-curvature solution in the rightmost one.

\section{Some Special Cases}

The goal of this section is to show two special cases exhibiting a relation between the Dubejko-weighted random walk on an infinite circle-packed triangulation and the parabolicity of its carrier. In Proposition 3.1, whose arguments appear in [16] and [15] but are provided here for completeness, we show that if the carrier is bounded then the WRW is transient. In Proposition 3.5 we show that if the carrier is the entire plane then the WRW is recurrent.

Proposition 3.1. Let $\Omega \subseteq \mathbb{R}^{2}$ be a bounded domain and $\left\{C_{v}\right\}_{v \in V}$ a circle-packed infinite triangulation of $\Omega$. Then the Dubejko-weighted random walk on $\left\{C_{v}\right\}_{v \in V}$ is transient.

Proof. Let $\left(X_{n}\right)_{n \in \mathbb{N}}$ be the weighted random walk started at some vertex $X_{0} \equiv \rho$. By Theorem 2.4, $\left(X_{n}\right)_{n \in \mathbb{N}}$ is a martingale. A bounded martingale converges almost surely to some random variable (see for example [5]), and so $X_{n} \stackrel{a . s}{\longrightarrow} Y$ for some random variable $Y$. Choose any two vertices in the graph, say $\rho$ and $\rho^{\prime}$. Assume towards contradiction that the random walk is recurrent, then $\rho$ and $\rho^{\prime}$ are almost surely both visited infinitely often. Now, $X_{n}$ converges to $Y$ a.s and $X_{n}=\rho$ infinitely often a.s, which implies that $Y=\rho$ almost surely. A similar argument shows that $Y=\rho^{\prime}$ almost surely. But $\rho \neq \rho^{\prime}$ in contradiction.

The proof of Proposition 3.1 was immediate from martingale arguments. For Proposition 3.5, we will use extensively the theory of probability and electric networks. For background on this field one may read chapter 2 of [12].

Definition 3.2. Let $(G, c)$ be a network. The Dirichlet energy of a function $f: V(G) \rightarrow \mathbb{R}$ is defined by

$$
\mathcal{E}(f):=\sum_{u v \in E(G)} c_{u v}(f(v)-f(u))^{2} .
$$

We will need the following criterion for recurrence which follows from Dirichlet's Principle (see exercise 2.93 of [12]):

Proposition 3.3. Let $(G, c)$ be a network. Write $G=(V, E)$ and fix some $\rho \in V$. Then $(G, c)$ is recurrent iff for any $\varepsilon>0$ there exists a finitely supported function $f: V \rightarrow \mathbb{R}$ with $f(\rho)=1$ and $\mathcal{E}(f)<\varepsilon$.

In the case that the carrier is the entire plane, the following Lemma shows that the effective resistance across any well-chosen annulus in the plane is at least a constant, which will be shown later to imply recurrence.

Lemma 3.4. Let $\left\{C_{v}\right\}_{v \in V}$ be a circle-packed infinite triangulation of the entire plane. Then there exists some $C>0$ such that for any $R>0$ there exists some finitely supported $f: V \rightarrow \mathbb{R}$ such that $\left.f\right|_{V \cap B(0, R)} \equiv 1$ and $\mathcal{E}(f) \leq C$. 
Proof. Assume WLOG that $R>0$ is large enough such that if $0 \in C_{v}$ for some $v \in V$ then $C_{v} \subseteq B(0, R)$.

Define a continuous $\phi: \mathbb{R}^{2} \rightarrow \mathbb{R}$ in polar coordinates by:

$$
\phi(r, \theta)= \begin{cases}1, & r \leq R \\ \frac{2 R-r}{R}, & R \leq r \leq 2 R . \\ 0, & r \geq 2 R\end{cases}
$$

$\phi$ induces a function $f$ on $V$ by assigning to a vertex $v \in V$ the value that $\phi$ takes on the center of $C_{v}$. It is immediate that $\left.f\right|_{V \cap B(0, R)} \equiv 1$. In addition, $f$ is finitely supported since its support is contained in the compact subset $\overline{B(0,2 R)}$ of the carrier, which can intersect only finitely many polygons of $\left\{C_{v}\right\}_{v \in V}$ (rigorously proven later in Lemma 5.14) and hence only finitely many vertices of $V$.

We move on to bound the Dirichlet energy of $f$. Let $G=(V, E)$ be the tangency graph of $\left\{C_{v}\right\}_{v \in V}$. For each edge $u v \in E$ there is an orthodiagonal quadrilateral $Q_{u v}$ whose diagonals are $e$ and $e^{\dagger}$, as shown in Figure 3.1.

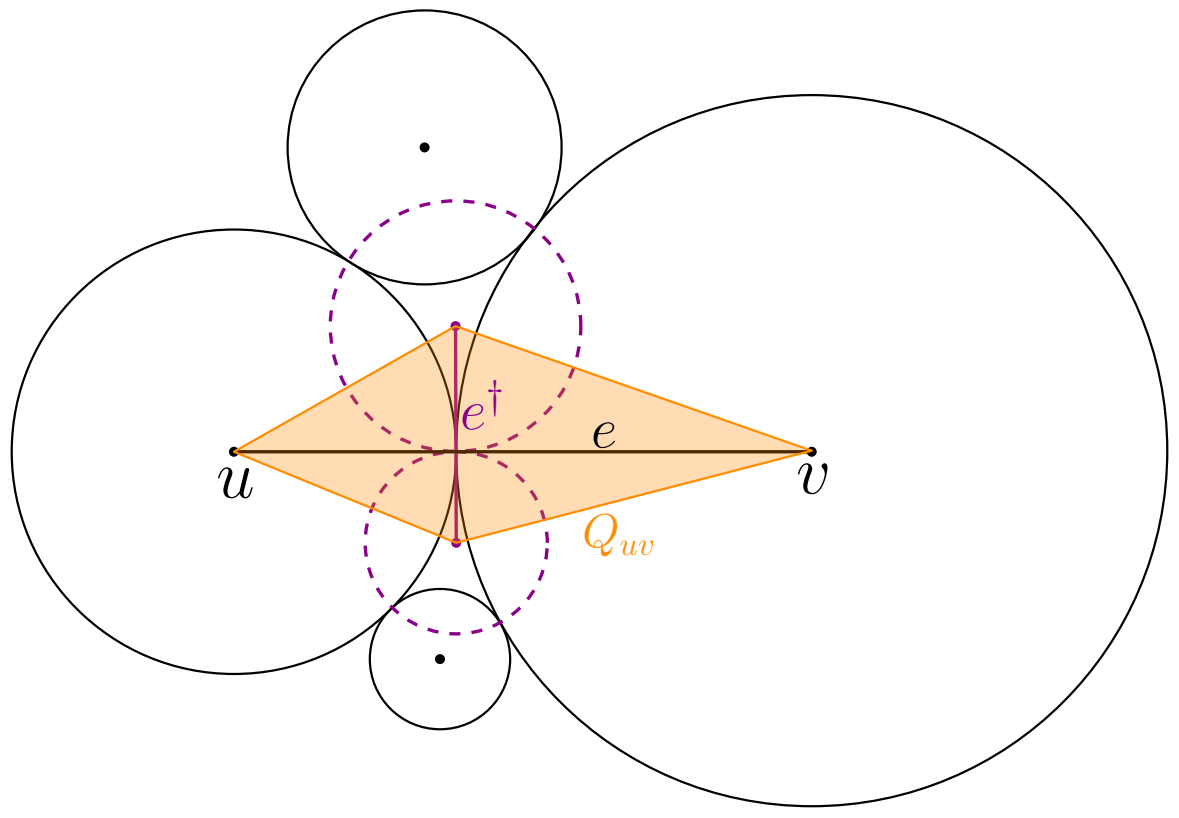

FiguRE 3.1. The orthodiagonal quadrilateral $Q_{u v}$.

Using these quadrilaterals we divide the energy into two parts, bounding each separately: Set $E_{1}=\left\{e \in E \mid Q_{u v} \subseteq B(0,8 R)\right\}$ and $E_{2}=E \backslash E_{1}$, and for each $i \in\{1,2\}$ set $\mathcal{E}_{i}=\sum_{u v \in E_{i}}(f(u)-f(v))^{2} c_{u v}$. Then $\mathcal{E}(f)=\mathcal{E}_{1}+\mathcal{E}_{2}$.

For the first part, notice that $\phi$ is $\frac{1}{R}$-Lipschitz since the gradient's norm is:

$$
\|\nabla \phi(r, \theta)\|= \begin{cases}\frac{1}{R}, & R<r<2 R \\ 0, & \text { otherwise }\end{cases}
$$

and so for each $e=u v \in E$ we can bound:

$$
(f(u)-f(v))^{2} c_{u v} \leq\left(\frac{1}{R}|e|\right)^{2} \frac{\left|e^{\dagger}\right|}{|e|}=\frac{2}{R^{2}} \operatorname{Area}\left(Q_{e}\right) .
$$

Since the quadrilaterals $\left\{Q_{e}\right\}_{e \in E_{1}}$ have disjoint interiors and are contained in $B(0,8 R)$ we have:

$$
\mathcal{E}_{1} \leq \sum_{e \in E_{1}} \frac{2}{R^{2}} \operatorname{Area}\left(Q_{e}\right)=\frac{2}{R^{2}} \operatorname{Area}\left(\bigcup_{e \in E_{1}} Q_{e}\right) \leq \frac{2}{R^{2}} \cdot \operatorname{Area}(B(0,8 R))=128 \pi .
$$

We move on to bound the second term $\mathcal{E}_{2}$. Let $W=\left\{v \in V: C_{v} \cap B(0,4 R) \neq \emptyset\right.$ and $\left.r_{v} \geq 4 R\right\}$, where $r_{v}$ is the radius of $C_{v}$. 
We first claim that the cardinality of $W$ is bounded by some constant number (say, 16). An easy way to show this is through area considerations: If $v \in W$ then there exists some point $p \in C_{v} \cap B(0,4 R)$. This implies that there exists some $q \in B(0,6 R)$ such that $\|q-v\| \leq r_{v}-2 R$ : Indeed, set $t=\max \left\{0,1-\frac{r_{v}-2 R}{\|p-v\|}\right\}$, and then $q=p+t(v-p)$ can be shown to satisfy the above conditions. By the choice of $q$ we have $B(q, 2 R) \subseteq C_{v} \cap B(0,8 R)$, and hence:

$$
|W| \cdot 4 R^{2} \leq \sum_{v \in W} \operatorname{Area}\left(C_{v} \cap B(0,8 R)\right)=\text { Area }\left(\bigcup_{v \in W} C_{v} \cap B(0,8 R)\right) \leq \operatorname{Area}(B(0,8 R))=64 R^{2} .
$$

Dividing both sides by $4 R^{2}$ shows that $|W| \leq 16$.

We next claim that if $e=u v \in E_{2}$ and $e$ has some energy contribution then $u \in W$ or $v \in W$ : If $f(u)=f(v)=0$ then the energy contribution is zero, so we can assume WLOG that $f(u) \neq 0$, and so $u \in B(0,2 R)$. We will show that in this case $v \in W$. First, we have $r_{u}<2 R$, because if we assume otherwise we have $0 \in C_{u}$ and by the choice of $R$ we get $C_{u} \subseteq B(0, R)$ in contradiction. Thus, we have $C_{u} \subseteq B(0,4 R)$ and since $C_{v}$ touches $C_{u}$ we deduce $C_{v} \cap B(0,4 R) \neq \emptyset$. Next, The quadrilateral $Q_{e}$ is convex, so from $Q_{e} \nsubseteq \subseteq B(0,8 R)$ we deduce that one of its vertices lies outside $B(0,8 R)$. This vertex cannot be $u$ since $u \in B(0,2 R)$. If the vertex is $v$, we have

$$
r_{v}=\left(r_{v}+r_{u}\right)-r_{u}=\|v-u\|-r_{u} \geq\|v\|-\|u\|-r_{u}>8 R-2 R-2 R=4 R,
$$

so $v \in W$ as needed. Otherwise, another vertex of the quadrilateral which we denote by $z$ lies outside $B(0,8 R)$, and let $w \in V$ be the vertex such that uvw is the face whose incenter is $z$. Let $r$ be the inradius of uvw. Since $z \notin B(0,8 R)$ and $\underline{u \in B(0,2 R)}$ we have:

$$
\|z-u\| \geq\|z\|-\|u\|>8 R-2 R=6 R .
$$

On the other hand, using Lemma 2.7 and the orthogonality of $e$ and $e^{\dagger}$ we get:

$$
\|z-u\|=\sqrt{r_{u}^{2}+r^{2}}<\sqrt{r_{u}^{2}+r_{u} r_{v}}<\sqrt{4 R^{2}+2 R \cdot r_{v}}
$$

Combining the two inequalities involving $\|z-u\|$ and solving for $r_{v}$ gives $r_{v}>16 R$, which finishes the second claim about $W$.

Finally, using the trivial bound $0 \leq f \leq 1$ together Lemma 2.11 and what we know about $W$, we obtain:

$$
\mathcal{E}_{2} \leq \sum_{v \in W} \sum_{u \sim v}(f(u)-f(v))^{2} c_{u v} \leq \sum_{v \in W} \sum_{u \sim v} 1 \cdot c_{u v} \leq \sum_{v \in W} 2 \pi \leq 32 \pi .
$$

In total we get $\mathcal{E}(f)=\mathcal{E}_{1}+\mathcal{E}_{2} \leq C$ for $C=160 \pi$.

Proposition 3.5. Let $\left\{C_{v}\right\}_{v \in V}$ be a circle-packed infinite triangulation of the entire plane. Then the Dubejkoweighted random walk on $\left\{C_{v}\right\}_{v \in V}$ is recurrent.

Proof. Let $G=(V, E)$ be the tangency graph of $\left\{C_{v}\right\}_{v \in V}$ and fix some $\rho \in V$. Construct a sequence of radii $R_{1}<R_{2}<\ldots$ and a sequence of finitely supported functions $f_{i}: V \rightarrow \mathbb{R}$ in the following way: Choose $R_{1}>0$ such that $\rho \in B\left(0, R_{1}\right)$, and choose $f_{1}$ to be a finitely supported function as in Lemma 3.4 such that $\mathcal{E}\left(f_{1}\right)<C$ and $\left.f_{1}\right|_{V \cap B\left(0, R_{1}\right)} \equiv 1$. For each $i>1$, assume we've defined $R_{i}>0$ and $f_{i}$. Since $f_{i}$ is finitely supported and $G$ is locally finite, we can choose some $R_{i+1}>R_{i}$ such that for every $v \notin B\left(0, R_{i+1}\right)$ we have that $f_{i}$ vanishes on $v$ and on all its neighbours. Applying Lemma 3.4 again, there exists some finitely supported $f_{i+1}: V \rightarrow \mathbb{R}$ with $\mathcal{E}\left(f_{i+1}\right)<C$ and $\left.f_{i+1}\right|_{V \cap B\left(0, R_{i+1}\right)} \equiv 1$.

For each $N$ set $g_{N}:=\sum_{i=1}^{N} \frac{1}{N} f_{i}$. Then $g_{N}$ is finitely supported (as the sum of finitely many finitely supported functions). Furthermore, since $f_{i}(\rho)=1$ for every $1 \leq i \leq N$, we have $g_{N}(\rho)=1$ as their mean. Using Proposition 3.3 , it is enough to show that $\lim _{N \rightarrow \infty} \mathcal{E}\left(g_{N}\right)=0$ in order to deduce recurrence.

To this end, we claim that for every $u v \in E$ there is at most one $1 \leq i \leq N$ such that $f_{i}(u) \neq f_{i}(v)$. Indeed, assume $f_{i}(u) \neq f_{i}(v)$. then at least one of these is nonzero. By the construction of $f_{i}$, this implies that $u, v \in B\left(0, R_{i+1}\right)$. Thus, for any $j>i$ we have $\left.f_{j}\right|_{V \cap B\left(0, R_{j}\right)} \equiv 1$ and in particular $f_{j}(u)=f_{j}(v)=1$. Similarly, $f_{i}(u)$ and $f_{i}(v)$ cannot 
both be equal to 1 , and WLOG we may assume that $f_{i}(u) \neq 1$. Then by construction of $f_{i}$ we have $u \notin B\left(0, R_{i}\right)$, and so for each $j<i$ we have $u \notin B\left(0, R_{j+1}\right)$ and by the choice of $R_{j+1}$ we deduce $f_{j}(u)=f_{j}(v)=0$.

Now, having established that each edge $u v \in E$ contributes to the energy of at most one $f_{i}$, the energy $\mathcal{E}\left(g_{N}\right)$ decomposes as follows:

$$
\mathcal{E}\left(g_{N}\right)=\mathcal{E}\left(\sum_{i=1}^{N} \frac{1}{N} f_{i}\right)=\sum_{i=1}^{N} \mathcal{E}\left(\frac{1}{N} f_{i}\right)=\sum_{i=1}^{N} \frac{1}{N^{2}} \mathcal{E}\left(f_{i}\right)<\sum_{i=1}^{N} \frac{1}{N^{2}} \cdot C=\frac{C}{N},
$$

and so $\lim _{N \rightarrow \infty} \mathcal{E}\left(g_{N}\right)=0$ as needed.

\section{Some Integration Lemmas}

The goal of this section is to prove Lemmas 4.8 and 4.10, providing bounds on integrals involving harmonic functions over polygons whose angles are not too sharp.

Definition 4.1. Let $U \subseteq \mathbb{R}^{2}$ be open. A function $f: U \rightarrow \mathbb{R}$ is said to be harmonic in $U$ if it is twice continuously differentiable and for every $x_{0} \in U$ the Laplacian vanishes on $x_{0}$, i.e $\left(\frac{\partial^{2} f}{\partial x^{2}}+\frac{\partial^{2} f}{\partial y^{2}}\right)\left(x_{0}\right)=0$.

We begin be recalling the following classical inequality (for a proof see [1] for example):

Theorem 4.2. (Harnack's Inequality): Let $f$ be nonnegative and harmonic in $B\left(x_{0}, R\right)$. Let $x \in B\left(x_{0}, R\right)$ and set $r=\left\|x-x_{0}\right\|$. Then:

$$
\frac{R-r}{R+r} f\left(x_{0}\right) \leq f(x) \leq \frac{R+r}{R-r} f\left(x_{0}\right) .
$$

Lemma 4.3. Let $f$ be nonnegative and harmonic in $B\left(x_{0}, R\right)$. Then:

$$
\left\|\nabla f\left(x_{0}\right)\right\| \leq \frac{2 f\left(x_{0}\right)}{R} .
$$

Proof. Let $x \in B\left(x_{0}, R\right)$, and write $r=\left\|x-x_{0}\right\|$. By Harnack's inequality we have:

$$
\frac{R-r}{R+r} f\left(x_{0}\right) \leq f(x) \leq \frac{R+r}{R-r} f\left(x_{0}\right) .
$$

Rearranging this we get:

$$
-\frac{2}{R+r} f\left(x_{0}\right) \leq \frac{f(x)-f\left(x_{0}\right)}{r} \leq \frac{2}{R-r} f\left(x_{0}\right) .
$$

Using the inequality $R-r \leq R+r$, we deduce:

$$
\frac{\left|f(x)-f\left(x_{0}\right)\right|}{r} \leq \frac{2}{R-r} f\left(x_{0}\right) .
$$

If $\nabla f\left(x_{0}\right)=0$ then the inequality holds. Otherwise, Since $\left\|\nabla f\left(x_{0}\right)\right\|$ is the directional derivative in the direction of $\hat{u}=\frac{\nabla f\left(x_{0}\right)}{\left\|\nabla f\left(x_{0}\right)\right\|}$, we have:

$$
\left\|\nabla f\left(x_{0}\right)\right\|=\lim _{h \rightarrow 0}\left|\frac{f\left(x_{0}+h \hat{u}\right)-f\left(x_{0}\right)}{h}\right| \leq \limsup _{h \rightarrow 0} \frac{2}{R-h} f\left(x_{0}\right)=\frac{2}{R} f\left(x_{0}\right) .
$$

Lemma 4.4. Let $f$ be harmonic in $B\left(x_{0}, R\right)$ and continuous in $\overline{B\left(x_{0}, R\right)}$. Then:

$$
\left\|\nabla f\left(x_{0}\right)\right\| \leq \frac{2}{R}\left(\max _{x \in \overline{B\left(x_{0}, R\right)}} f(x)-\min _{x \in \overline{B\left(x_{0}, R\right)}} f(x)\right) .
$$

Proof. Set $g=f-\min _{x \in \overline{B\left(x_{0}, R\right)}} f(x)$. Then $g$ is nonnegative and harmonic in $B\left(x_{0}, R\right)$, and applying Lemma 4.3 gives:

$$
\left\|\nabla f\left(x_{0}\right)\right\|=\left\|\nabla g\left(x_{0}\right)\right\| \leq \frac{2}{R} g\left(x_{0}\right) \leq \max _{x \in \overline{B\left(x_{0}, R\right)}} f(x)-\min _{x \in \overline{B\left(x_{0}, R\right)}} f(x) .
$$


Lemma 4.5. There exists some $C_{0}>0$ such that for every $R>0, x_{0} \in \mathbb{R}^{2}$ and $f$ harmonic in $B\left(x_{0}, R\right)$ and continuous in $\overline{B\left(x_{0}, R\right)}$ we have in polar coordinates:

$$
\int_{0}^{2 \pi} \int_{0}^{R}\left\|\nabla f\left(x_{0}+r e^{i \theta}\right)\right\|^{2} R d r d \theta \leq C_{0} \int_{0}^{2 \pi} \int_{0}^{R}\left\|\nabla f\left(x_{0}+r e^{i \theta}\right)\right\|^{2} r d r d \theta .
$$

Proof. Notice first that both the LHS and the RHS integrals are invariant under translations and dilations of the domain. Explicitly, defining $\tilde{f}: \overline{B(0,1)} \rightarrow \mathbb{R}$ by $\tilde{f}(x)=f\left(x_{0}+R x\right)$ we have $\nabla \tilde{f}(x)=R \nabla f\left(x_{0}+R x\right)$, and so for the LHS:

$$
\int_{0}^{2 \pi} \int_{0}^{1}\left\|\nabla \tilde{f}\left(r e^{i \theta}\right)\right\|^{2} d r d \theta=\int_{0}^{2 \pi} \int_{0}^{1}\left\|\nabla f\left(x_{0}+R r e^{i \theta}\right)\right\|^{2} R^{2} d r d \theta=\int_{0}^{2 \pi} \int_{0}^{R}\left\|\nabla f\left(x_{0}+r e^{i \theta}\right)\right\|^{2} R d r d \theta .
$$

Similarly, for the RHS:

$$
C_{0} \int_{0}^{2 \pi} \int_{0}^{1}\left\|\nabla \tilde{f}\left(r e^{i \theta}\right)\right\|^{2} r d r d \theta=C_{0} \int_{0}^{2 \pi} \int_{0}^{1}\left\|\nabla f\left(x_{0}+R r e^{i \theta}\right)\right\|^{2} R^{2} r d r d \theta=C_{0} \int_{0}^{2 \pi} \int_{0}^{R}\left\|\nabla f\left(x_{0}+r e^{i \theta}\right)\right\|^{2} r d r d \theta .
$$

Thus we may assume WLOG that $R=1$ and $x_{0}=0$. We present two proofs for the claim: the first using Harnack's inequality and the second using Fourier analysis.

For the first proof, set $\varepsilon_{d}$ to be the RHS (or the Dirichlet energy), $\varepsilon_{d}=\int_{0}^{2 \pi} \int_{0}^{1}\left\|\nabla f\left(r e^{i \theta}\right)\right\|^{2} r d r d \theta$, and assume that for some $C_{0}>0$ we have:

$$
\int_{0}^{2 \pi} \int_{0}^{1}\left\|\nabla f\left(r e^{i \theta}\right)\right\|^{2} d r d \theta>C_{0} \cdot \varepsilon_{d}
$$

Since $\int_{0}^{2 \pi} \int_{\frac{1}{4}}^{1}\left\|\nabla f\left(r e^{i \theta}\right)\right\|^{2} d r d \theta \leq \int_{0}^{2 \pi} \int_{\frac{1}{4}}^{1}\left\|\nabla f\left(r e^{i \theta}\right)\right\|^{2} \cdot 4 r \cdot d r d \theta \leq 4 \varepsilon_{d}$, we have:

$$
C_{0} \cdot \varepsilon_{d}<\int_{0}^{2 \pi} \int_{0}^{\frac{1}{4}}\left\|\nabla f\left(r e^{i \theta}\right)\right\|^{2} d r d \theta+4 \varepsilon_{d}
$$

so we find that:

$$
\int_{0}^{2 \pi} \int_{0}^{\frac{1}{4}}\left\|\nabla f\left(r e^{i \theta}\right)\right\|^{2} d r d \theta>\left(C_{0}-4\right) \varepsilon_{d} .
$$

Therefore, there exists some $x_{0} \in B\left(0, \frac{1}{4}\right)$ such that $\left\|\nabla f\left(x_{0}\right)\right\|^{2}>\frac{4\left(C_{0}-4\right)}{2 \pi} \varepsilon_{d}$. By Lemma 4.4 applied to $f$ on $\overline{B\left(x_{0}, \frac{1}{4}\right)}$ we deduce that:

$$
\left(\max _{x \in B\left(x_{0}, \frac{1}{4}\right)} f(x)-\min _{x \in B\left(x_{0}, \frac{1}{4}\right)} f(x)\right)^{2} \geq \frac{\left(\frac{1}{4}\right)^{2}}{4} \cdot\left\|\nabla f\left(x_{0}\right)\right\|^{2}>\frac{1}{64} \cdot \frac{4\left(C_{0}-4\right)}{2 \pi} \varepsilon_{d} .
$$

Fix some $1>r \geq \frac{1}{2}$. By the maximum and minimum principles for harmonic functions, $f$ restricted to $\overline{B(0, r)}$ achieves its maximum and minimum on the boundary $\partial B(0, r)$. Thus, by inequality 4.1 and the fact that $\overline{B\left(x_{0}, \frac{1}{4}\right) \subseteq}$ $\overline{B(0, r)}$ we get that:

$$
\left(\max _{x \in \partial B(0, r)} f(x)-\min _{x \in \partial B(0, r)} f(x)\right)^{2}>\frac{1}{64} \cdot \frac{4\left(C_{0}-4\right)}{2 \pi} \varepsilon_{d} .
$$

Let $\theta_{1}, \theta_{2}$ be angles such that:

$$
f\left(r e^{i \theta_{1}}\right)=\min _{x \in \partial B(0, r)} f(x), \quad f\left(r e^{i \theta_{2}}\right)=\max _{x \in \partial B(0, r)} f(x) .
$$


Let $c$ be the shorter circular arc with center at 0 going from $r e^{i \theta_{1}}$ to $r e^{i \theta_{2}}$. We use Cauchy-Schwartz for line integrals to get:

$$
\left(f\left(r e^{i \theta_{2}}\right)-f\left(r e^{i \theta_{1}}\right)\right)^{2}=\left(\int_{c} \nabla f \cdot d \underline{r}\right)^{2} \leq\left(\int_{c}\|\nabla f\|^{2} d s\right) \cdot \operatorname{length}(c) \leq\left(\int_{\partial B(0, r)}\|\nabla f\|^{2} d s\right) \cdot \pi r
$$

And so:

$$
\int_{0}^{2 \pi}\left\|\nabla f\left(r e^{i \theta}\right)\right\|^{2} r d \theta>\frac{1}{\pi r} \cdot\left(\frac{1}{64} \cdot \frac{4\left(C_{0}-4\right)}{2 \pi} \varepsilon_{d}\right)=\frac{\left(C_{0}-4\right)}{32 \pi^{2} r} \cdot \varepsilon_{d}
$$

Finally we get:

$$
\begin{aligned}
\varepsilon_{d} & =\int_{0}^{\frac{1}{2}} \int_{0}^{2 \pi}\left\|\nabla f\left(r e^{i \theta}\right)\right\|^{2} r d \theta d r+\int_{\frac{1}{2}}^{1} \int_{0}^{2 \pi}\left\|\nabla f\left(r e^{i \theta}\right)\right\|^{2} r d \theta d r \geq \\
& \geq \int_{\frac{1}{2}}^{1} \int_{0}^{2 \pi}\left\|\nabla f\left(r e^{i \theta}\right)\right\|^{2} r d \theta d r>\int_{\frac{1}{2}}^{1} \frac{\left(C_{0}-4\right)}{32 \pi^{2} r} \cdot \varepsilon_{d} d r=\frac{\left(C_{0}-4\right) \ln 2}{32 \pi^{2}} \cdot \varepsilon_{d} .
\end{aligned}
$$

This implies $C_{0} \leq 4+\frac{32 \pi^{2}}{\ln 2}$, and the Lemma follows for any value of $C_{0}$ larger than $4+\frac{32 \pi^{2}}{\ln 2}$.

For the second proof, assume that $f$ is smooth on the boundary. Write the Fourier expansion of $f$ restricted to the unit circle: $f(1, \theta)=a_{0}+\sum_{n=1}^{\infty}\left(a_{n} \cos (n \theta)+b_{n} \sin (n \theta)\right)$. Then we have:

$$
f\left(r e^{i \theta}\right)=a_{0}+\sum_{n=1}^{\infty} r^{n}\left(a_{n} \cos (n \theta)+b_{n} \sin (n \theta)\right) .
$$

The gradient is:

$$
\begin{aligned}
\nabla f\left(r e^{i \theta}\right) & =\frac{\partial f}{\partial r} \cdot \hat{r}+\frac{1}{r} \frac{\partial f}{\partial \theta} \cdot \hat{\theta}= \\
& =\left(\sum_{n=1}^{\infty} n r^{n-1}\left(a_{n} \cos (n \theta)+b_{n} \sin (n \theta)\right)\right) \hat{r}+\left(\sum_{n=1}^{\infty} n r^{n-1}\left(-a_{n} \sin (n \theta)+b_{n} \cos (n \theta)\right)\right) \hat{\theta}
\end{aligned}
$$

Using the orthogonality of $\hat{r}, \hat{\theta}$ we find:

$$
\begin{aligned}
\left\|\nabla f\left(r e^{i \theta}\right)\right\|^{2} & =\left(\sum_{n=1}^{\infty} n r^{n-1}\left(a_{n} \cos (n \theta)+b_{n} \sin (n \theta)\right)\right)^{2}+\left(\sum_{n=1}^{\infty} n r^{n-1}\left(-a_{n} \sin (n \theta)+b_{n} \cos (n \theta)\right)\right)^{2}= \\
& =\sum_{n, m=1}^{\infty} n m r^{n+m-2}\left(a_{n} \cos (n \theta)+b_{n} \sin (n \theta)\right)\left(a_{m} \cos (m \theta)+b_{m} \sin (m \theta)\right)+ \\
& +\sum_{n, m=1}^{\infty} n m r^{n+m-2}\left(-a_{n} \sin (n \theta)+b_{n} \cos (n \theta)\right)\left(-a_{m} \sin (m \theta)+b_{m} \cos (m \theta)\right) .
\end{aligned}
$$

We integrate on theta first, and use the orthogonality of the Fourier basis:

$$
\begin{aligned}
\int_{0}^{2 \pi}\left\|\nabla f\left(r e^{i \theta}\right)\right\|^{2} d \theta & =\sum_{n=1}^{\infty} n^{2} r^{2 n-2}\left(a_{n}^{2} \cdot \pi+b_{n}^{2} \cdot \pi\right)+\sum_{n=1}^{\infty} n^{2} r^{2 n-2}\left(a_{n}^{2} \cdot \pi+b_{n}^{2} \cdot \pi\right)= \\
& =2 \pi \sum_{n=1}^{\infty} n^{2} r^{2 n-2}\left(a_{n}^{2}+b_{n}^{2}\right) .
\end{aligned}
$$


Now the LHS of the Lemma's statement is:

$$
\int_{0}^{1} \int_{0}^{2 \pi}\left\|\nabla f\left(r e^{i \theta}\right)\right\|^{2} d \theta d r=\int_{0}^{1}\left(2 \pi \sum_{n=1}^{\infty} n^{2} r^{2 n-2}\left(a_{n}^{2}+b_{n}^{2}\right)\right) d r=\sum_{n=1}^{\infty} \frac{2 \pi n^{2}}{2 n-1}\left(a_{n}^{2}+b_{n}^{2}\right) .
$$

The RHS is:

$$
\int_{0}^{1} \int_{0}^{2 \pi}\left\|\nabla f\left(r e^{i \theta}\right)\right\|^{2} d \theta \cdot r d r=\int_{0}^{1}\left(2 \pi \sum_{n=1}^{\infty} n^{2} r^{2 n-1}\left(a_{n}^{2}+b_{n}^{2}\right)\right) d r=\sum_{n=1}^{\infty} \frac{2 \pi n^{2}}{2 n}\left(a_{n}^{2}+b_{n}^{2}\right) .
$$

For each $n \in \mathbb{N}$ we have $\frac{1}{2 n-1} \leq \frac{2}{2 n}$, so taking $C_{0}=2$ finishes the proof.

Definition 4.6. For any $\alpha>0$, a polygon $P$ is called $\alpha$-nice if all of its angles are larger than $\alpha$.

Definition 4.7. A polygon $P$ is called tangential if there is a circle tangent to all of its sides.

Lemma 4.8. For every angle $\alpha>0$ there exists some $C_{1}(\alpha)>0$ such that for every $\alpha$-nice tangential polygon $P$ and for every $f$ continuous in $P$ and harmonic in the interior of $P$ we have:

$$
\int_{\partial P} \int_{T_{a z}}\|\nabla f(w)\|^{2} d w d z \leq C_{1}(\alpha) \cdot \iint_{P}\|\nabla f\|^{2} d A
$$

where $a$ is the center of the circle inscribed in $P$ and $T_{a z}$ is the straight line segment connecting a to $z$.

Proof. Both sides of the inequality are invariant under translations and dilations of $P$. Therefore, we may assume that $a=0$ and that the radius of the inscribed circle is 1 . Let $\theta_{1} \leq \theta_{2} \leq \ldots \leq \theta_{n}$ be the angles of the tangency points of the unit circle and the sides of the polygon. Set $\theta_{n+1}=\theta_{1}$ for ease of notation. For each $1 \leq i \leq n$, write $\varphi_{i}=\frac{1}{2}\left(\theta_{i+1}-\theta_{i}\right)$ as in Figure 4.1 .

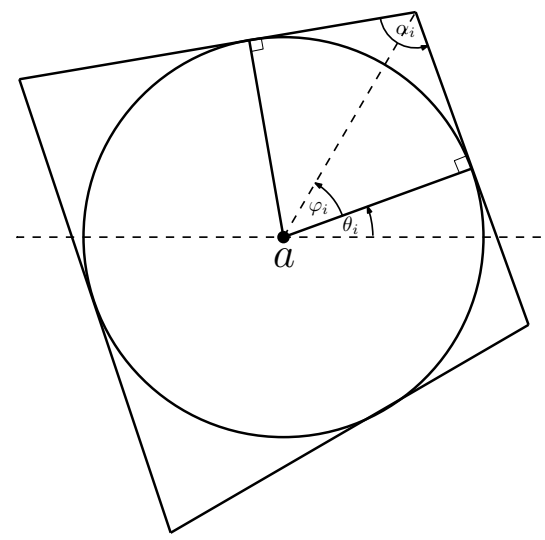

Figure 4.1. Tangential polygon and its incircle

The boundary $\partial P$ of the polygon is given in polar coordinates by:

$$
r(\theta)=\left\{\begin{array}{ll}
\frac{1}{\cos \left(\theta-\theta_{i}\right)}, & 0 \leq \theta-\theta_{i} \leq \varphi_{i} \\
\frac{1}{\cos \left(\theta_{i+1}-\theta\right)} & 0 \leq \theta_{i+1}-\theta \leq \varphi_{i}
\end{array} \quad \Longrightarrow \quad \frac{d r}{d \theta}=\left\{\begin{array}{ll}
\frac{\sin \left(\theta-\theta_{i}\right)}{\cos ^{2}\left(\theta-\theta_{i}\right)}, & 0 \leq \theta-\theta_{i} \leq \varphi_{i} \\
-\frac{\sin \left(\theta_{i+1}-\theta\right)}{\cos ^{2}\left(\theta_{i+1}-\theta\right)} & 0 \leq \theta_{i+1}-\theta \leq \varphi_{i}
\end{array} .\right.\right.
$$

The length element is $d s=\sqrt{r^{2}+\left(\frac{d r}{d \theta}\right)^{2}} d \theta$, and letting $\alpha_{i}=\pi-2 \varphi_{i}$ be the $i$-th angle of the polygon, we bound this factor:

$$
\sqrt{r^{2}+\left(\frac{d r}{d \theta}\right)^{2}}=\left\{\begin{array}{ll}
\frac{1}{\cos ^{2}\left(\theta-\theta_{i}\right)}, & 0 \leq \theta-\theta_{i} \leq \varphi_{i} \\
\frac{1}{\cos ^{2}\left(\theta_{i+1}-\theta\right)}, & 0 \leq \theta_{i+1}-\theta \leq \varphi_{i}
\end{array} \leq \frac{1}{\cos ^{2}\left(\varphi_{i}\right)}=\frac{1}{\sin ^{2}\left(\frac{\alpha_{i}}{2}\right)} \leq \frac{1}{\sin ^{2}\left(\frac{\alpha}{2}\right)}\right.
$$


Setting $C_{1}^{\prime}=\frac{1}{\sin ^{2}\left(\frac{\alpha}{2}\right)}$, we bound the integral:

$$
\begin{aligned}
\int_{\partial P} \int_{T_{0, z}}\|\nabla f(w)\|^{2} d w d z & =\int_{0}^{2 \pi}\left(\int_{0}^{r(\theta)}\left\|\nabla f\left(r e^{i \theta}\right)\right\|^{2} d r\right) \sqrt{r(\theta)^{2}+\left(\frac{d r(\theta)}{d \theta}\right)^{2}} d \theta \leq \\
& \leq C_{1}^{\prime} \cdot \int_{0}^{2 \pi} \int_{0}^{r(\theta)}\left\|\nabla f\left(r e^{i \theta}\right)\right\|^{2} d r d \theta
\end{aligned}
$$

We now divide the polygon into two regions and bound the integral on each of them: The first region is the unit disk $\mathbb{D}$, on which we use Lemma 4.5 to get:

$$
\underbrace{\int_{0}^{2 \pi} \int_{0}^{1}\left\|\nabla f\left(r e^{i \theta}\right)\right\|^{2} d r d \theta}_{I_{1}} \leq C_{0} \iint_{\mathbb{D}}\|\nabla f\|^{2} d A \leq C_{0} \iint_{P}\|\nabla f\|^{2} d A .
$$

For the remaining region we have $r \geq 1$ so we get:

$$
\underbrace{\int_{0}^{2 \pi} \int_{1}^{r(\theta)}\left\|\nabla f\left(r e^{i \theta}\right)\right\|^{2} d r d \theta}_{I_{2}} \leq \int_{0}^{2 \pi} \int_{1}^{r(\theta)}\left\|\nabla f\left(r e^{i \theta}\right)\right\|^{2} \cdot r d r d \theta=\iint_{P \backslash \mathbb{D}}\|\nabla f\|^{2} d A \leq \iint_{P}\|\nabla f\|^{2} d A .
$$

Setting $C_{1}=C_{1}^{\prime}\left(1+C_{0}\right)$ we finish the proof:

$$
\begin{aligned}
\int_{\partial P} \int_{T_{0, z}}\|\nabla f(w)\|^{2} d w d z & \leq C_{1}^{\prime} \cdot \int_{0}^{2 \pi} \int_{0}^{r(\theta)}\left\|\nabla f\left(r \cdot e^{i \theta}\right)\right\|^{2} d r d \theta=C_{1}^{\prime} \cdot\left(I_{1}+I_{2}\right) \leq \\
& \leq C_{1}^{\prime}\left(C_{0}+1\right) \iint_{P}\|\nabla f\|^{2} d A=C_{1} \iint_{P}\|\nabla f\|^{2} d A .
\end{aligned}
$$

Definition 4.9. Given a polygon $P$ and a side $S$ of $\partial P$, we say that $S$ is an $\alpha$-nice side of $P$ if both angles incident to $S$ are larger than $\alpha$.

Lemma 4.10. Let $0<\alpha<\pi$. Then in the notation of Lemma 4.8 and using the same constant $C_{1}(\alpha)$, for every $P$ tangential polygon, $S_{1}, S_{2}, \ldots, S_{k}$ distinct $\alpha$-nice sides of $P$ and $f$ continuous on $P$ and harmonic in the interior of $P$ we have:

$$
\sum_{j=1}^{k} \int_{S_{j}} \int_{T_{a z}}\|\nabla f(w)\|^{2} d w d z \leq C_{1}(\alpha) \cdot \iint_{P}\|\nabla f\|^{2} d A
$$

Proof. We prove this by finding an $\alpha$-nice tangential polygon $P^{\prime} \subseteq P$. Consider a vertex $L$ of $\partial P$ with angle $\beta$. If $\beta>\alpha$ we are done. Otherwise, we replace $L$ with two vertices $L_{1}, L_{2}$ in the following way, illustrated in Figure 4.2 . Denote by $X$ the intersection of the line segment $a L$ and the incircle. Pass a tangent to the circle at $X$, and denote by $L_{1}, L_{2}$ the intersection points of this line with the two tangents from $L$ to the circle. For each $i \in\{1,2\}$, the angle facing the circle at $L_{i}$ is $\frac{1}{2} \beta+\frac{1}{2} \pi$ since it is an exterior angle to the triangle $L X L_{i}$ which has remote interior angles $\frac{1}{2} \beta$ and $\frac{1}{2} \pi$. If $\frac{1}{2} \beta+\frac{\pi}{2}>\alpha$ we are done. Otherwise, perform this step again on each of $L_{1}, L_{2}$ and repeat. After $n$ steps the angle is $\frac{1}{2^{n}} \beta+\left(1-\frac{1}{2^{n}}\right) \pi$ and since $\alpha<\pi$ and $\lim _{n \rightarrow \infty}\left(\frac{1}{2^{n}} \beta+\left(1-\frac{1}{2^{n}}\right) \pi\right)=\pi$, for $n$ large enough we will have an angle larger than $\alpha$. Perform this process on each vertex of $\partial P$ to get an $\alpha$-nice tangential polygon $P^{\prime} \subseteq P$. Notice that all the tangency points of $P$ are also tangency points in $P^{\prime}$. 


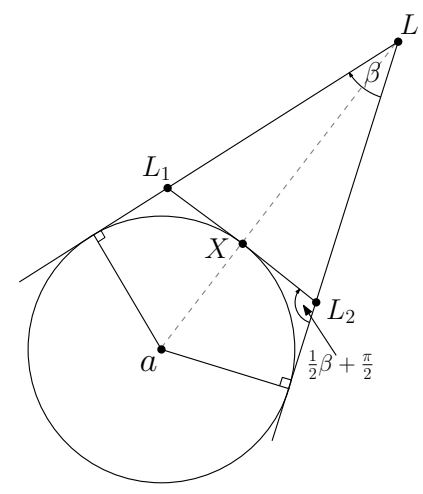

Figure 4.2. Polygon vertex replacement process.

For every $i$, the two vertices incident to the side $S_{i}$ had angles larger than $\alpha$ to begin with, so $S_{i}$ is also a side of $\partial P^{\prime}$, and thus:

$$
\sum_{i=1}^{k} \int_{S_{i}} \int_{T_{a z}}\|\nabla f(w)\|^{2} d w d z \leq \int_{\partial P^{\prime}} \int_{T_{a z}}\|\nabla f(w)\|^{2} d w d z .
$$

$f$ is continuous on $P^{\prime}$ and harmonic in its interior because $P^{\prime} \subseteq P$, so using Lemma 4.8 on $P^{\prime}$ we get:

$$
\int_{\partial P^{\prime}} \int_{T_{a z}}\|\nabla f(w)\|^{2} d w d z \leq C_{1}(\alpha) \cdot \iint_{P^{\prime}}\|\nabla f\|^{2} d A .
$$

Finally, since $P^{\prime} \subseteq P$ :

$$
C_{1}(\alpha) \cdot \iint_{P^{\prime}}\|\nabla f\|^{2} d A \leq C_{1}(\alpha) \cdot \iint_{P}\|\nabla f\|^{2} d A .
$$

Putting together the three inequalities above we are done.

\section{The General Case}

5.1. A Useful Coupling. The goal of this subsection is to prove Corollary 5.9, which says that given a circlepacked infinite triangulation, we can add under certain conditions circles to the packing such that the weighted random walks on the original and new circle packings can be coupled. We begin with a few Lemmas about reversible Markov chains.

Definition 5.1. A Markov chain on a state space $V$ is said to be represented by a network $(G, c)$ where $G$ has vertex set $V$ if the chain and the weighted random walk on $(G, c)$ share the same transition matrix.

Note that in Definition 1.1 of a network, the set of edges $E$ is somewhat superfluous: It is sometimes more convenient to compactly represent the network by the pair $(V, c)$ where $c: V \times V \rightarrow[0, \infty)$ is extended to take the value 0 on pairs of vertices not in $E$, and the edge set $E$ can be recovered as the support of $c$.

The weighted random walk on a network $(G, c)$ is an irreducible reversible Markov chain: Irreducibility follows from the requirement that $G$ be connected, and the sum $\pi(v)$ of the weights around a vertex $v$ is easily seen to be a reversible measure. Conversely, every irreducible reversible Markov chain can be represented by some network: Indeed, given a reversible positive measure $\pi: V \rightarrow(0, \infty)$ on the states, a possible weight function is $c_{u v}=\pi(u) P_{u, v}$, where $P$ is the chain's transition matrix. The weights of the representing network are only unique up to a multiplicative constant. See chapter 2 of [12] for some background on reversible Markov chains.

Definition 5.2. Let $\left(X_{n}\right)_{n \in \mathbb{N}}$ be a Markov chain on a state space $V$, and let $W \subseteq V$. The random sequence $\left(Y_{n}\right)_{n \in \mathbb{N}}$ obtained from $\left(X_{n}\right)_{n \in \mathbb{N}}$ by deleting all appearances of $W$ is called the Markov chain censored to $V \backslash W$. 
The subset $W$ usually has the property that the event $\left\{\exists N\right.$ such that $\left.\forall n \geq N, X_{n} \in W\right\}$ has probability zero so that the sequence $\left(Y_{n}\right)_{n \in \mathbb{N}}$ is almost surely an infinite sequence. In particular, this is the case when the chain is irreducible and $W$ is a finite proper subset of $V$. The censored chain (also known as the watched chain) is itself a Markov chain, as shown in Lemma 6-6 of [10]. The following Lemma shows that censoring also preserves reversibility, and tells us how to transform the corresponding network when censoring a single state:

Lemma 5.3. Let $(V, c)$ be a network and let $V^{\prime}:=V \backslash\{w\}$ for some $w \in V$. Then the censoring to $V^{\prime}$ of the weighed random walk on $(V, c)$ is a reversible Markov chain represented by the network $\left(V^{\prime}, c^{\prime}\right)$, where $c^{\prime}: V^{\prime} \times V^{\prime} \rightarrow[0, \infty)$ is given by $c_{x y}^{\prime}=c_{x y}+\frac{c_{x w} c_{y w}}{\pi(w)-c_{w w}}$.

Proof. We need to show that the weighted random walk on $\left(V^{\prime}, c^{\prime}\right)$ satisfies the transition probabilities of the censored chain. Let $x, y \in V^{\prime}$. Denote by $\left(X_{n}\right)_{n \in \mathbb{N}}$ and $\left(Y_{n}\right)_{n \in \mathbb{N}}$ the original and censored chains respectively. The transition probabilities of the censored network are:

$$
\begin{aligned}
\mathbb{P}_{x}\left(Y_{1}=y\right) & =\mathbb{P}_{x}\left(X_{1}=y\right)+\sum_{n=1}^{\infty} \mathbb{P}_{x}\left(X_{1}=X_{2}=\ldots=X_{n}=w, X_{n+1}=y\right)= \\
& =\frac{c_{x y}}{\pi(x)}+\sum_{n=1}^{\infty} \frac{c_{x w}}{\pi(x)} \cdot\left(\frac{c_{w w}}{\pi(w)}\right)^{n-1} \cdot \frac{c_{w y}}{\pi(w)}=\frac{1}{\pi(x)}\left(c_{x y}+\frac{c_{x w} c_{w y}}{\pi(w)} \frac{1}{1-\frac{c_{w w}}{\pi(w)}}\right)= \\
& =\frac{c_{x y}^{\prime}}{\pi(x)} .
\end{aligned}
$$

We next claim that the sum of weights $\pi^{\prime}(x)$ around a vertex $x$ in the new network $\left(V^{\prime}, c^{\prime}\right)$ is the same as in the old network. Indeed,

$$
\pi^{\prime}(x)=\sum_{y \in V^{\prime}} c_{x y}^{\prime}=\left(\sum_{y \in V^{\prime}} c_{x y}\right)+\frac{c_{x w}}{\pi(w)-c_{w w}} \sum_{y \in V^{\prime}} c_{y w}=\left(\sum_{y \in V^{\prime}} c_{x y}\right)+c_{x w}=\pi(x) .
$$

Putting the equalities above together we get $\mathbb{P}_{x}\left(Y_{1}=y\right)=\frac{c_{x y}^{\prime}}{\pi^{\prime}(x)}$ as needed.

Remark 5.4. Some network reduction techniques, such as the $Y-\Delta$ transform and resistors in series, are special cases of Lemma 5.3.

Corollary 5.5. Let $(V, c)$ be a network and let $V^{\prime}:=V \backslash W$ for some finite proper subset $W \subseteq V$. Then the censoring to $V^{\prime}$ of the weighed random walk on $(V, c)$ is reversible and is represented by a network $\left(V^{\prime}, c^{\prime}\right)$ such that for every $x, y \in V^{\prime}$ that are not both in $\partial W=\left\{v \in V^{\prime} \mid \exists w \in W, c_{v w}>0\right\}$ we have $c_{x y}^{\prime}=c_{x y}$.

Proof. Write $W=\left\{w_{1}, w_{2}, \ldots, w_{n}\right\}$. The censored chain to $V^{\prime}$ is exactly the chain obtained by censoring the states in $W$ one after the other. Hence, by applying Lemma $5.3 n$ times, the chain censored to $V^{\prime}$ is reversible. For the second part, let $x, y \in V^{\prime}$ with $x \notin \partial W$. Then for every $i \in\{1,2, \ldots, n\}$ we have $c_{x w_{i}}=0$, and hence the weight $c_{x y}$ is unchanged in each application of Lemma 5.3.

When censoring a chain to $V \backslash W$, we may be adding loops at vertices of $\partial W$. The following Lemma shows that deleting repetitions from the chain corresponds to deleting loops from the representing network:

Definition 5.6. Let $\left(X_{n}\right)_{n \in \mathbb{N}}$ be an irreducible Markov chain. Its induced repetition-deleted chain is the random sequence obtained from $\left(X_{n}\right)_{n \in \mathbb{N}}$ by replacing consecutive appearances of the same state with a single appearance. Formally, set $\tau_{0}=0$ and for every $i>0$ set $\tau_{i}=\inf \left\{n>\tau_{i-1} \mid X_{n} \neq X_{\tau_{i-1}}\right\}$. Then the repetition-deleted chain induced by $\left(X_{n}\right)_{n \in \mathbb{N}}$ is $\left(X_{\tau_{i}}\right)_{i \in \mathbb{N}}$.

Lemma 5.7. Let $\left(X_{n}\right)_{n \in \mathbb{N}}$ be a Markov chain represented by a network $(V, c)$. Then the repetition-deleted chain $\left(Y_{n}\right)_{n \in \mathbb{N}}$ induced by $\left(X_{n}\right)_{n \in \mathbb{N}}$ is reversible. Furthermore, a network $\left(V, c^{\prime}\right)$ representing $\left(Y_{n}\right)_{n \in \mathbb{N}}$ is obtained from $(V, c)$ by deleting self-loops. 
Proof. The new weights $c^{\prime}$ are given by:

$$
c_{x y}^{\prime}= \begin{cases}c_{x y}, & x \neq y \\ 0, & x=y\end{cases}
$$

and so the new sum of weights around a vertex $x$ is:

$$
\pi^{\prime}(x)=\sum_{y \in V} c_{x y}^{\prime}=\left(\sum_{y \in V} c_{x y}\right)-c_{x x}=\pi(x)-c_{x x} .
$$

We need to show that the weighted random walk on $\left(V, c^{\prime}\right)$ satisfies the transition probabilities of the repetitiondeleted chain. Indeed, if $y \neq x$ then:

$$
\begin{aligned}
\mathbb{P}_{x}\left(Y_{1}=y\right) & =\sum_{n=0}^{\infty} \mathbb{P}_{x}\left(X_{0}=X_{1}=\ldots=X_{n}=x, X_{n+1}=y\right)= \\
& =\sum_{n=0}^{\infty}\left(\frac{c_{x x}}{\pi(x)}\right)^{n} \cdot \frac{c_{x y}}{\pi(x)}=\frac{c_{x y}}{\pi(x)} \cdot \frac{1}{1-\frac{c_{x x}}{\pi(x)}}=\frac{c_{x y}}{\pi(x)-c_{x x}}=\frac{c_{x y}^{\prime}}{\pi^{\prime}(x)} .
\end{aligned}
$$

Otherwise, $y=x$ and then by the definition of $\left(Y_{n}\right)_{n \in \mathbb{N}}$ we have $\mathbb{P}_{x}\left(Y_{1}=x\right)=0=\frac{c_{x x}^{\prime}}{\pi^{\prime}(x)}$.

The following Lemma shows that when removing the set of circles contained in a triangle of an infinite circlepacked triangulation, if this set is finite then the original weighted random walk and the weighted random walk can be coupled:

Lemma 5.8. Let $\left\{C_{v}\right\}_{v \in V}$ be a circle-packed infinite triangulation. Let xyz be a triangle in the graph such that the set $W \subseteq V$ of the vertices contained in the interior of xyz is finite, as in Figure 5.1. Let $\left(X_{n}\right)_{n \in \mathbb{N}}$ be a Dubejkoweighted random walk on $\left\{C_{v}\right\}_{v \in V}$ and let $\left(Y_{n}\right)_{n \in \mathbb{N}}$ be the chain obtained from $\left(X_{n}\right)_{n \in \mathbb{N}}$ by first censoring it to $V^{\prime}=V \backslash W$ and then deleting repetitions. Then $\left(Y_{n}\right)_{n \in \mathbb{N}}$ is a Dubejko-weighted random walk on $\left\{C_{v}\right\}_{v \in V^{\prime}}$.

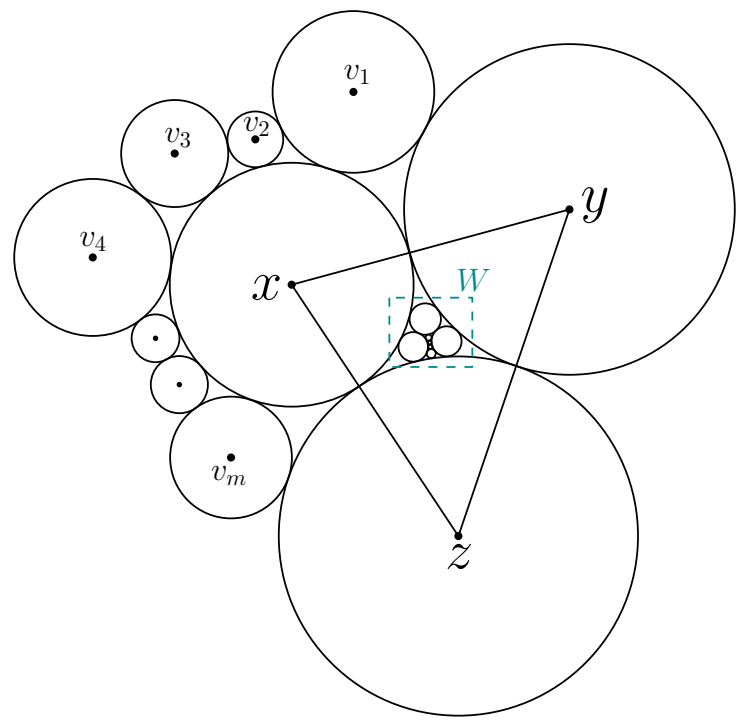

FIGURE 5.1. Triangle $x y z$, the set $W$ of vertices contained in the interior of $x y z$ and the neighbours of $x$.

Proof. Let $G=(V, E)$ and $G^{\prime}=\left(V^{\prime}, E^{\prime}\right)$ be the respective tangency graphs of $\left\{C_{v}\right\}_{v \in V}$ and $\left\{C_{v}\right\}_{v \in V^{\prime}}$, and let $c$ and $c^{\prime}$ be their respective Dubejko weights. We first notice that if $u_{1}, u_{2} \in V^{\prime}$ are not both in $\{x, y, z\}$ then $c_{u_{1}, u_{2}}^{\prime}=c_{u_{1}, u_{2}}$ : Indeed, if $u_{1} u_{2} \notin E$ then $u_{1} u_{2} \notin E^{\prime}$ and both sides of the equation are zero, and otherwise equality holds as well since both the edge $u_{1} u_{2}$ and its dual have not changed in length when removing the circles corresponding to vertices of $W$.

Next, note that $\partial W \subseteq\{x, y, z\}$ (in fact equality holds if $W$ is nonempty). Thus, by Corollary 5.5 and Lemma 
5.7, $\left(Y_{n}\right)_{n \in \mathbb{N}}$ is a reversible Markov chain represented by a network $\left(V^{\prime}, c^{\prime \prime}\right)$ with no self-loops such that for all $u_{1}, u_{2} \in V^{\prime}$ that are not both in $\{x, y, z\}$ we have $c_{u_{1}, u_{2}}^{\prime \prime}=c_{u_{1}, u_{2}}$.

We need to show that $c^{\prime \prime} \equiv c^{\prime}$. For $u_{1}, u_{2} \in V^{\prime}$ that are not both in $\{x, y, z\}$ we've already seen that $c_{u_{1}, u_{2}}^{\prime}=c_{u_{1}, u_{2}}=$ $c_{u_{1}, u_{2}}^{\prime \prime}$. So we are left with showing that $c^{\prime \prime}$ and $c^{\prime}$ agree on edges of the triangle $x y z$. Denote the neighbours of $x$ that are not in $W$ by $y, v_{1}, v_{2}, \ldots, v_{m}, z$, as in Figure 5.1, and suppose $X_{0} \equiv x$. For any vertex $u \in V$, let $\tau_{u}:=\min \left\{n \geq 0: X_{n}=u\right\}$. Set a stopping time

$$
\tau=\min \left\{\tau_{y}, \tau_{v_{1}}, \tau_{v_{2}}, \ldots, \tau_{v_{m}}, \tau_{z}\right\} .
$$

$\tau$ is almost surely finite since $\{\tau=\infty\}$ is the event that $\left(X_{n}\right)_{n \in \mathbb{N}}$ stays in the finite set of vertices $W$ forever. For any $u \in\left\{y, v_{1}, v_{2}, \ldots, v_{m}, z\right\}$, the event $\left\{\tau=\tau_{u}\right\}$ is exactly the event $\left\{Y_{1}=u\right\}$. By Theorem 2.4, the sequence $\left(X_{n}\right)_{n \in \mathbb{N}}$ is a martingale. Stopping this martingale at stopping time $\tau$ yields a bounded martingale, in which case Doob's optional stopping theorem implies:

$$
x=\mathbb{P}_{x}\left(Y_{1}=y\right) \cdot y+\mathbb{P}_{x}\left(Y_{1}=z\right) \cdot z+\sum_{j=1}^{m} \mathbb{P}_{x}\left(Y_{1}=v_{j}\right) \cdot v_{j}
$$

or, passing $x$ to the other side:

$$
\mathbb{P}_{x}\left(Y_{1}=y\right) \cdot(y-x)+\mathbb{P}_{x}\left(Y_{1}=z\right) \cdot(z-x)+\sum_{j=1}^{m} \mathbb{P}_{x}\left(Y_{1}=v_{j}\right) \cdot\left(v_{j}-x\right)=0
$$

Now, substituting $\mathbb{P}_{x}\left(Y_{1}=u\right)=\frac{c_{x u}^{\prime \prime}}{\pi^{\prime \prime}(x)}$ and multiplying the equation by $\pi^{\prime \prime}(x)$ we get:

$$
c_{x y}^{\prime \prime}(y-x)+c_{x z}^{\prime \prime}(z-x)+\sum_{j=1}^{m} c_{x v_{j}}^{\prime \prime}\left(v_{j}-x\right)=0
$$

For every $j \in\{1,2, \ldots, m\}$, since $v_{j} \notin \partial W$, we have an equality $c_{x v_{j}}^{\prime \prime}=c_{x v_{j}}$, and thus:

$$
c_{x y}^{\prime \prime}(y-x)+c_{x z}^{\prime \prime}(z-x)=-\sum_{j=1}^{m} c_{x v_{j}}\left(v_{j}-x\right) .
$$

Start a Dubejko-weighted random walk $\left(W_{n}\right)_{n \in \mathbb{N}}$ on $\left\{C_{v}\right\}_{v \in V^{\prime}}$ at $W_{0} \equiv x$. By Theorem 2.4 again, we have that $\left(W_{n}\right)_{n \in \mathbb{N}}$ is a martingale, and in particular $\mathbb{E}\left[W_{0}\right]=\mathbb{E}\left[W_{1}\right]$. A similar calculation to the one we just did, substituting $\mathbb{P}_{x}\left(W_{1}=u\right)=\frac{c_{x u}^{\prime}}{\pi^{\prime}(x)}$, multiplying by $\pi^{\prime}(x)$ and using $c_{x v_{j}}^{\prime}=c_{x v_{j}}$, gives us:

$$
c_{x y}^{\prime}(y-x)+c_{x z}^{\prime}(z-x)=-\sum_{j=1}^{m} c_{x v_{j}}\left(v_{j}-x\right) .
$$

Combining equations 5.1 and 5.2 yields:

$$
c_{x y}^{\prime \prime}(y-x)+c_{x z}^{\prime \prime}(z-x)=c_{x y}^{\prime}(y-x)+c_{x z}^{\prime}(z-x) .
$$

$y-x$ and $z-x$ are two sides of a triangle and hence independent vectors in $\mathbb{R}^{2}$. Thus, $c_{x y}^{\prime \prime}=c_{x y}^{\prime}$ and $c_{x z}^{\prime \prime}=c_{x z}^{\prime}$ as needed. A similar calculation with $y$ as center circle instead of $x$ shows that $c_{y z}^{\prime \prime}=c_{y z}^{\prime}$.

The following Corollary is an extension of Lemma 5.8 which allows us, under certain conditions, to remove an infinite amount of vertices from the packing:

Corollary 5.9. Let $\left\{C_{v}\right\}_{v \in V}$ be a circle-packed infinite triangulation with tangency graph $G$. Let $\left\{x_{i} y_{i} z_{i}\right\}_{i \in I}$ for some $I=\{1,2, . ., N\}$ or $I=\mathbb{N}$ be a family of triangles in $G$ with pairwise disjoint interiors such that for each $i \in I$, the set of vertices $W_{i}$ contained in the interior of the triangle $x_{i} y_{i} z_{i}$ is finite. Set $W=\bigcup_{i \in I} W_{i}$ and $V^{\prime}=V \backslash W$. For some $\rho \in V^{\prime}$, start a Dubejko-weighted random walk $\left(X_{n}\right)_{n \in \mathbb{N}}$ on $\left\{C_{v}\right\}_{v \in V}$ at $\rho$. Then the chain $\left(Y_{n}\right)_{n \in \mathbb{N}}$ obtained from $\left(X_{n}\right)_{n \in \mathbb{N}}$ by first censoring it to $V^{\prime}$ and then deleting repetitions is a Dubejko-weighted random walk 
on $\left\{C_{v}\right\}_{v \in V^{\prime}}$ started at $\rho$. In particular, this coupling implies that the weighted random walks on $\left\{C_{v}\right\}_{v \in V}$ and $\left\{C_{v}\right\}_{v \in V^{\prime}}$ are either both recurrent or both transient.

Proof. We start by showing that the process $\left(Y_{n}\right)_{n \in \mathbb{N}}$ is well-defined in the sense that it is almost surely an infinite sequence.

We first claim that for each $i \in I$ we have $x_{i}, y_{i}, z_{i} \in V^{\prime}$ : Suppose otherwise, then WLOG $x_{i} \in W_{j}$ for some $j$. Since $x_{i} \notin W_{i}$, we have $j \neq i$, and so $x_{i} \in \operatorname{int}\left(\operatorname{conv}\left\{x_{j}, y_{j}, z_{j}\right\}\right)$. This implies that some small ball around $x_{i}$ is contained in int $\left\{\operatorname{conv}\left\{x_{j}, y_{j}, z_{j}\right\}\right\}$. But this ball also intersects the interior of $\operatorname{conv}\left\{x_{i}, y_{i}, z_{i}\right\}$, in contradiction to the assumption of disjoint interiors for the triangles. Thus, in the graph induced on $W$, each connected component is contained in some $W_{i}$, and in particular each connected component is finite.

We next claim that for every $m \in \mathbb{N}$, the event $A_{m}=\left\{\exists t>m\right.$ such that $X_{t} \in V^{\prime}$ and $\left.X_{t} \neq X_{m}\right\}$ has probability 1 . Since its complement is contained in the countable union $\bigcup_{v \in V} B_{m, v}$ for $B_{m, v}=\left\{X_{m}=v \wedge\left((\forall t>m) X_{t} \in W \cup\{v\}\right)\right\}$, it is enough to show that $\mathbb{P}_{\rho}\left(B_{m, v}\right)=0$. The connected component of $v$ in $W \cup\{v\}$ is finite because $v$ has finite degree and so its addition to $W$ can only join together finitely many components of $W$, which are all finite. Thus, the event $B_{m, v}$ implies that after time $m$, the random walker never leaves the finite component of $v$ in $W \cup\{v\}$, so indeed $B_{m, v}$ has probability zero.

Finally, the event $A=\bigcup_{m \in \mathbb{N}} A_{m}$ has probability 1 since it is the countable union of such events. But $A$ implies that the result $\left(Y_{n}\right)_{n \in \mathbb{N}}$ of censoring $W$ and deleting repetitions is an infinite sequence since $A$ guarantees that infinitely often the random walker has moved between different states of $V^{\prime}$. Thus, $\left(Y_{n}\right)_{n \in \mathbb{N}}$ is an infinite sequence with probability 1 .

We move to show that $\left(Y_{n}\right)_{n \in \mathbb{N}}$ is a Dubejko-weighted random walk on $\left\{C_{v}\right\}_{v \in V^{\prime}}$ : Set $\left(Y_{n}^{0}\right)_{n \in \mathbb{N}}$ to be $\left(X_{n}\right)_{n \in \mathbb{N}}$, and for each $i \in I$ let $\left(Y_{n}^{i}\right)_{n \in \mathbb{N}}$ be obtained from $\left(Y_{n}^{i-1}\right)_{n \in \mathbb{N}}$ by censoring the vertices of $W_{i}$ and then deleting repetitions. Fix some $k \in \mathbb{N}$. We claim that there exists some deterministic $t_{k} \in \mathbb{N}$ such that $\left(Y_{0}, Y_{1}, \ldots, Y_{k}\right)=\left(Y_{0}^{t_{k}}, Y_{1}^{t_{k}}, \ldots, Y_{k}^{t_{k}}\right)$. A key observation here is that for every $j$, the vertices in $\partial W_{j}=\left\{x_{j}, y_{j}, z_{j}\right\}$ are neighbours to each other, and so $Y_{i+1}$ has to be a neighbour of $Y_{i}$ in $G$ for every $i$. Hence, the first $k$ steps $Y_{0}, Y_{1}, \ldots, Y_{k}$ are contained in a ball $B_{k}$ of graph-radius $k$ around $\rho$ in the graph induced on $V^{\prime}$. Let $I_{k}=\left\{i \in I \mid\left\{x_{i}, y_{i}, z_{i}\right\} \cap B_{k} \neq \emptyset\right\}$. Locally finiteness of $G$ implies that $I_{k} \subseteq\left\{1,2, \ldots, t_{k}\right\}$ for some $t_{k} \in \mathbb{N}$, and so $\left(Y_{0}, Y_{1}, \ldots, Y_{k}\right)=\left(Y_{0}^{t_{k}}, Y_{1}^{t_{k}}, \ldots, Y_{k}^{t_{k}}\right)$. Now, By Lemma 5.8 applied $t_{k}$ times, $\left(Y_{n}^{t_{k}}\right)_{n \in \mathbb{N}}$ is a weighted random walk on $\left\{C_{v}\right\}_{v \in V \backslash \cup_{i=1}^{t_{k}} W_{i}}$. But the first $k$ steps in the weighted random walk on $\left\{C_{v}\right\}_{v \in V \backslash \cup_{i=1}^{t_{k} W_{i}}}$ are equal in distribution to the first $k$ steps of the weighted random walk on $\left\{C_{v}\right\}_{v \in V^{\prime}}$ since the balls of graph-radius $k$ around $\rho$ in both networks are identical. Thus, $\left(Y_{0}, Y_{1}, \ldots, Y_{k}\right)$ also has the distribution of the first $k$ steps of the weighted random walk on $\left\{C_{v}\right\}_{v \in V^{\prime}}$, and since $k$ is arbitrary we get that $\left(Y_{n}\right)_{n \in \mathbb{N}}$ is a weighted random walk on $\left\{C_{v}\right\}_{v \in V^{\prime}}$ started at $\rho$.

Finally, we show that the random walks are either both recurrent or both transient. Assume first that $\left\{C_{v}\right\}_{v \in V}$ is recurrent. Then $\rho$ is visited infinitely often in $\left(X_{n}\right)_{n \in \mathbb{N}}$ with probability 1 . The event that $\rho$ appears infinitely many times in $\left(X_{n}\right)_{n \in \mathbb{N}}$ but only finitely many times in $\left(Y_{n}\right)_{n \in \mathbb{N}}$ is contained in the event $\bigcup_{m=1}^{\infty} B_{m, \rho}$ defined above, so it has probability zero. Thus, $\rho$ almost surely appears infinitely often in $\left(Y_{n}\right)_{n \in \mathbb{N}}$ and so $\left\{C_{v}\right\}_{v \in V^{\prime}}$ is recurrent. Now assume that $\left\{C_{v}\right\}_{v \in V}$ is transient. Then with positive probability $\rho$ appears finitely often in $\left(X_{n}\right)_{n \in \mathbb{N}}$, and hence finitely often in $\left(Y_{n}\right)_{n \in \mathbb{N}}$, implying that $\left\{C_{v}\right\}_{v \in V^{\prime}}$ is transient.

The following Proposition is not used in the proof of Theorem 1.3, but we've included it here because of the proof's resemblance to that of Lemma 5.8:

Proposition 5.10. Let $\left\{C_{v}\right\}_{v \in V}$ be a circle-packed infinite triangulation and $G=(V, E)$ its tangency graph. Let $x y z$ be a triangle in the graph such that the set $W$ of vertices contained in the interior of the triangle xyz is finite. Let $f: W \cup\{x, y, z\} \rightarrow \mathbb{R}$ be harmonic in $W$ with respect to the Dubejko weights of the circle packing. Then $f$ agrees with the unique affine function $g: \mathbb{R}^{2} \rightarrow \mathbb{R}$ taking the values $f(x), f(y), f(z)$ on $x, y, z$ respectively. 
Proof. Because $g$ is affine, its value on convex combinations of the form $a x+b y+c z$ for $a, b, c \geq 0$ with $a+b+c=1$ is given by:

$$
g(a x+b y+c z)=a f(x)+b f(y)+c f(z) .
$$

Let $w \in W \cup\{x, y, z\}$. Start a weighted random walk $\left(Z_{n}\right)_{n \in \mathbb{N}}$ at $Z_{0} \equiv w$, and stop it at $\tau=\min \left\{\tau_{x}, \tau_{y}, \tau_{z}\right\}$. By Theorem 2.4, $\left(Z_{n \wedge \tau}\right)_{n \in \mathbb{N}}$ is a bounded martingale. By the optional stopping theorem we deduce that $\mathbb{E} Z_{0}=\mathbb{E} Z_{\tau}$, so:

$$
w=\mathbb{P}_{w}\left(\tau=\tau_{x}\right) x+\mathbb{P}_{w}\left(\tau=\tau_{y}\right) y+\mathbb{P}_{w}\left(\tau=\tau_{z}\right) z .
$$

This presents $w$ (uniquely) as a convex combination of $\{x, y, z\}$. Since $f$ is harmonic, the sequence $\left(f\left(Z_{n \wedge \tau}\right)\right)_{n \in \mathbb{N}}$ is also a martingale. We apply the optional stopping theorem again on this martingale, which is also bounded since $W$ is finite, to get:

$$
\begin{aligned}
f(w) & =\mathbb{P}_{w}\left(\tau=\tau_{x}\right) f(x)+\mathbb{P}_{w}\left(\tau=\tau_{y}\right) f(y)+\mathbb{P}_{w}\left(\tau=\tau_{z}\right) f(z)= \\
& =g\left(\mathbb{P}_{w}\left(\tau=\tau_{x}\right) x+\mathbb{P}_{w}\left(\tau=\tau_{y}\right) y+\mathbb{P}_{w}\left(\tau=\tau_{z}\right) z\right)=g(w) .
\end{aligned}
$$

\subsection{Parabolicity.}

Definition 5.11. A domain $\Omega \subseteq \mathbb{R}^{2}$ is said to be parabolic if for every open set $U \subseteq \Omega$ and for any $x \in \Omega$, Brownian motion started at $x$ and killed at $\partial \Omega$ hits $U$ almost surely.

Definition 5.12. Let $\Omega \subseteq \mathbb{R}^{2}$ be a domain and $K \subseteq \Omega$ a compact set. The capacity of $K$ with respect to $\Omega$ is defined as:

$$
\operatorname{Cap}(K, \Omega)=\inf \iint_{\Omega}\|\nabla \phi\|^{2} d A,
$$

where the infimum is taken over all Lipschitz functions $\phi: \Omega \rightarrow \mathbb{R}$ with compact support in $\Omega$ such that $\left.\phi\right|_{K} \equiv 1$ and $0 \leq \phi \leq 1$. For a precompact open set $K$ in $\Omega$ we define $\operatorname{Cap}(K, \Omega)=\operatorname{Cap}(\bar{K}, \Omega)$.

The following equivalent condition for parabolicity, useful to us due to its resemblence to Proposition 3.3, is given in chapter 5 of [6]:

Proposition 5.13. A domain $\Omega \subseteq \mathbb{R}^{2}$ is parabolic iff the capacity of some/any precompact open set $K \subseteq \Omega$ is zero.

\subsection{Proof of Theorem 1.3.}

Lemma 5.14. Let $\left\{C_{v}\right\}_{v \in V}$ be a circle-packed infinite triangulation of a domain $\Omega \subseteq \mathbb{R}^{2}$, and let $A \subseteq \Omega$ be a compact subset. Then A intersects finitely many polygons of the packing.

Proof. Every point $x \in \Omega$ has a neighbourhood $U_{x} \subseteq \Omega$ which intersects at most 3 of the polygons: Since $\Omega=$ $\bigcup_{v \in V} P_{v}$, we know that $x \in P_{v}$ for some $v \in V$. If $x$ is in $\operatorname{int}\left(P_{v}\right)$ then $x$ is contained in exactly one polygon. The tangency graph of $\left\{C_{v}\right\}_{v \in V}$ is a triangulation, and hence the dual graph is 3-regular. Thus, if $x$ is a vertex of $\partial P_{v}$ whose sides are edges of the dual graph, then $x$ lies in exactly 3 polygons. Lastly, if $x$ is on $\partial P_{v}$ but is not a vertex then $x$ is in exactly 2 polygons. In all the cases above, a small enough ball around $x$ intersects at most 3 polygons. Since $A \subseteq \Omega=\bigcup_{x \in \Omega} U_{x}$, by compactness, there exists a finite set $x_{1}, x_{2}, \ldots, x_{m} \in \Omega$ such that $A \subseteq \bigcup_{i=1}^{m} U_{x_{i}}$. Because $U_{x_{i}}$ intersects at most 3 polygons, $A$ intersects at most $3 m$ polygons as needed.

Definition 5.15. Let $\left\{C_{v}\right\}_{v \in V}$ be a circle-packed infinite triangulation with tangency graph $G=(V, E)$, and let $\alpha>0$. An edge $e=u v \in E$ is said to be an $\alpha$-nice edge of $\left\{C_{v}\right\}_{v \in V}$ if its dual is an $\alpha$-nice side of both $P_{u}$ and $P_{v}$.

Lemma 5.16. Let $\left\{C_{v}\right\}_{v \in V}$ be a circle-packed infinite triangulation of some domain $\Omega \subseteq \mathbb{R}^{2}$ and denote its tangency graph by $G=(V, E)$. Fix some vertex $\rho \in V$. Then there exists some angle $\alpha_{0}>0$ such that for every $\varepsilon>0$, by 
inserting new circles to the packing, we can obtain a new circle-packed infinite triangulation $\left\{C_{v}\right\}_{v \in V^{\prime}}$ of $\Omega$ with tangency graph $G^{\prime}=\left(V^{\prime}, E^{\prime}\right)$ such that for the Dubejko-weighted networks $(G, c)$ and $\left(G^{\prime}, c^{\prime}\right)$ we have:

(1) Set $E_{2}^{\prime}=\left\{e^{\prime} \in E^{\prime} \mid e^{\prime}\right.$ is not $\left.\alpha_{0}-n i c e\right\}$. Then:

$$
\sum_{e^{\prime} \in E_{2}^{\prime}} c_{e^{\prime}}^{\prime} \leq \varepsilon
$$

(2) $\mathrm{C}_{\mathrm{eff}}(\rho \leftrightarrow \infty)=\mathrm{C}_{\mathrm{eff}}{ }^{\prime}(\rho \leftrightarrow \infty)$. In particular, $(G, c)$ is recurrent iff $\left(G^{\prime}, c^{\prime}\right)$ is recurrent.

Proof. Choose $\alpha_{0}$ to be some positive angle such that $\alpha_{0}<10^{-5}$ and such that all of the polygons of $\rho$ and its neighbours are $\alpha_{0}$-nice (this is possible by locally finiteness of $G$ ).

We insert circles in the following way: There is a countable amount of faces in the triangulation (since they have nonempty disjoint interiors). Enumerate them in some order $\left\{F_{n}\right\}_{n \in \mathbb{N}}$ and fix some $n \in \mathbb{N}$. Write $F_{n}=\{x, y, z\}$ for some $x, y, z \in V$ ordered by $r_{z} \geq r_{y} \geq r_{x}$, and denote by $M$ and $r$ the incenter and inradius of the triangle $x y z$. Denote the angles of $P_{x}, P_{y}, P_{z}$ at $M$ by $\alpha_{x}, \alpha_{y}, \alpha_{z}$. Note that for each $v \in\{x, y, z\}$ we have $\tan \frac{\alpha_{v}}{2}=\frac{r_{v}}{r}$ which implies $\tan \frac{\alpha_{z}}{2} \geq \tan \frac{\alpha_{y}}{2} \geq \tan \frac{\alpha_{x}}{2}$ and so $\alpha_{z} \geq \alpha_{y} \geq \alpha_{x}$. If $\alpha_{x}>\alpha_{0}$, insert no new circles, since in this case $\alpha_{z}, \alpha_{y}, \alpha_{x}>\alpha_{0}$. Otherwise, set $v_{0}=x$ and for each $i \in\{1,2, \ldots, k\}$, with $k=k(n)$ to be determined later, add a circle $C_{v_{i}}$ tangent to the three circles of $v_{i-1}, y, z$ and contained in the face $v_{i-1} y z$, effectively splitting this face into 3 faces. This process creates a chain of vertices $x=v_{0}, v_{1}, v_{2}, \ldots, v_{k}$ with respective radii $r_{x}=r_{0}, r_{1}, r_{2}, \ldots, r_{k}$ and with centers approaching the tangency point of $C_{y}$ and $C_{z}$, as in Figure 5.2. Denote the new edge weights by $c^{\prime}$ and the new polygons by $P^{\prime}$.

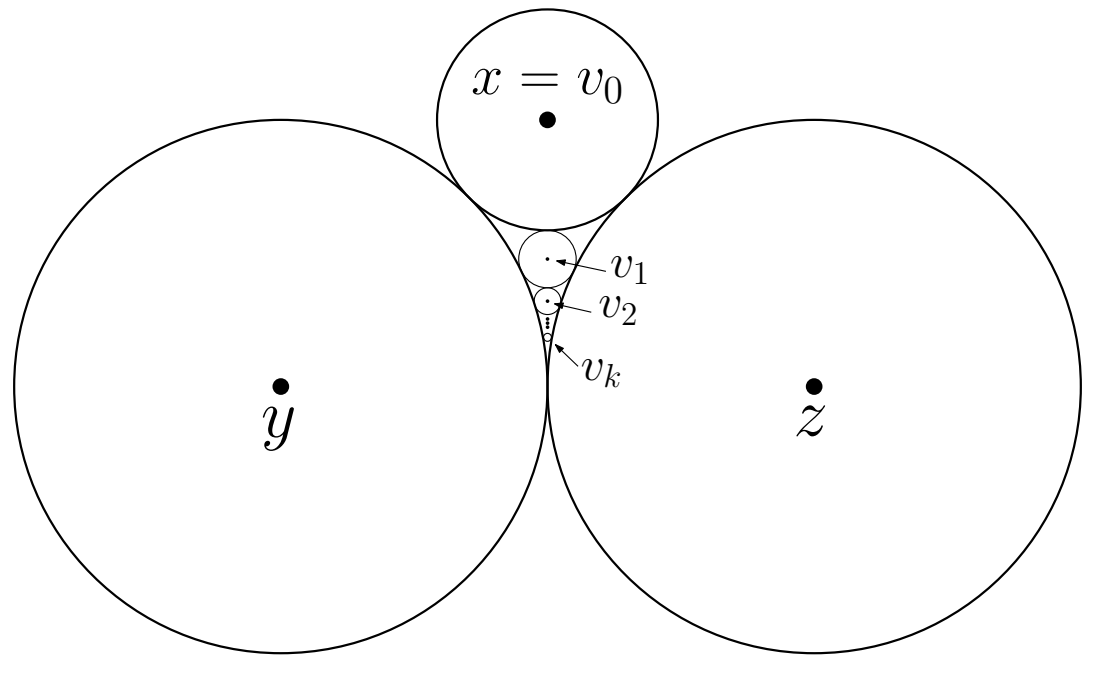

FIGURE 5.2. Circle insertion process

We first show that $k=k(n)$ can be chosen such that $c_{v_{k} y}^{\prime}+c_{v_{k} z}^{\prime}<\frac{\varepsilon}{2^{n}}$ : Since the circles $C_{v_{1}}, C_{v_{2}}, \ldots, C_{v_{k}}$ have disjoint interiors and are contained in the triangle $x y z$, we have:

$$
\sum_{i=1}^{k} \pi r_{i}^{2}=\sum_{i=1}^{k} \operatorname{Area}\left(C_{v_{i}}\right)=\operatorname{Area}\left(\bigcup_{i=1}^{k} C_{v_{i}}\right) \leq \operatorname{Area}\left(\triangle_{x y z}\right),
$$

so the series $\sum_{i=1}^{\infty} \pi r_{i}^{2}$ is bounded and in particular the sequence of terms $\pi r_{k}^{2}$ tends to zero, so $\lim _{k \rightarrow \infty} r_{k}=0$. Thus, by Proposition 2.6:

$$
c_{v_{k} y}^{\prime}=\frac{\sqrt{r_{k} r_{y}}}{r_{k}+r_{y}}\left(\sqrt{\frac{r_{z}}{r_{k}+r_{y}+r_{z}}}+\sqrt{\frac{r_{k-1}}{r_{k}+r_{y}+r_{k-1}}}\right) \leq \frac{\sqrt{r_{k} r_{y}}}{r_{k}+r_{y}} \cdot 2 \stackrel{k \rightarrow \infty}{\longrightarrow} 0 .
$$

A similar calculation replacing $y$ with $z$ shows that $\lim _{k \rightarrow \infty} c_{v_{k} z}^{\prime}=0$.

We next show that after the circle insertion process for the face $F_{n}=\{x, y, z\}$, out of all the incenters in the new 
faces created, the only angle that might not be $\alpha_{0}$-nice is the angle of $P_{v_{k}}^{\prime}$ at the incenter of $v_{k} y z$ : By Descartes' Theorem 2.14 for the four mutually-tangent circles with centers $y, z, v_{i}, v_{i+1}$ we get:

$$
\frac{1}{r_{i+1}}=\frac{1}{r_{y}}+\frac{1}{r_{z}}+\frac{1}{r_{i}}+2 \sqrt{\frac{1}{r_{y} r_{z}}+\frac{1}{r_{y} r_{i}}+\frac{1}{r_{z} r_{i}}},
$$

where we have chosen the plus sign in the formula above because $r_{i+1}$ is the smaller-radius solution. In particular, $\frac{1}{r_{i+1}} \geq \frac{1}{r_{i}}$ so $r_{i+1} \leq r_{i}$ and by induction for all $i$ we have $r_{i} \leq r_{0}=r_{x} \leq r_{y}, r_{z}$ and so:

$$
\frac{r_{i}}{r_{i+1}}=1+\frac{r_{i}}{r_{y}}+\frac{r_{i}}{r_{z}}+2 \sqrt{\frac{r_{i}^{2}}{r_{y} r_{z}}+\frac{r_{i}}{r_{y}}+\frac{r_{i}}{r_{z}}} \leq 3+2 \cdot \sqrt{3} \leq 7 .
$$

Denote by $Q_{i}$ the incenter of the triangle $y v_{i} v_{i+1}$ for any $i \in\{0,1, \ldots, k-1\}$. We claim that the respective angles $\alpha, \beta, \gamma$ of $P_{y}^{\prime}, P_{v_{i}}^{\prime}, P_{v_{i+1}}^{\prime}$ at $Q_{i}$ are larger than $\alpha_{0}$ : Indeed, since $\alpha_{0}<10^{-5}$ we can use Lemma 2.13 to deduce:

$$
\begin{aligned}
& \alpha>2 \tan ^{-1}\left(\sqrt{\frac{r_{y}}{r_{x}}}\right) \geq 2 \tan ^{-1}(1)=\frac{\pi}{2}>\alpha_{0} . \\
& \beta>2 \tan ^{-1}\left(\sqrt{\frac{r_{i}}{r_{i+1}}}\right) \geq 2 \tan ^{-1}(1)=\frac{\pi}{2}>\alpha_{0} . \\
& \gamma>2 \tan ^{-1}\left(\sqrt{\frac{r_{i+1}}{r_{i}}}\right) \geq 2 \tan ^{-1}\left(\sqrt{\frac{1}{7}}\right)>\alpha_{0} .
\end{aligned}
$$

A similar calculation with $z$ replacing $y$ shows that for every $i \in\{0,1, \ldots, k-1\}$ the angles of $P_{z}^{\prime}, P_{v_{i}}^{\prime}, P_{v_{i+1}}^{\prime}$ at the incenter of $z v_{i} v_{i+1}$ are larger than $\alpha_{0}$. Denote by $T$ the incenter of $v_{k} y z$. Denote by $\delta_{y}, \delta_{z}$ the respective angles of $P_{y}^{\prime}$ and $P_{z}^{\prime}$ at $T$. Then by Lemma 2.13 again we get for each $u \in\{y, z\}$ :

$$
\delta_{u}>2 \tan ^{-1}\left(\sqrt{\frac{r_{u}}{r_{k}}}\right) \geq 2 \tan ^{-1}(1)=\frac{\pi}{2}>\alpha_{0} .
$$

So indeed, out of the angles of new polygons contained in $x y z$, only the angle of $P_{v_{k}}^{\prime}$ at the incenter of $v_{k} y z$ might not be $\alpha_{0}$-nice.

For the first statement of the lemma, write $F_{n}=\left\{x_{n}, y_{n}, z_{n}\right\}$ as above for every $n \in \mathbb{N}$. Set $I \subseteq \mathbb{N}$ to be the set of indices of faces where we've inserted new circles. For every $i \in I$, let $s_{i} \in V^{\prime}$ be the vertex of the last circle inserted to $F_{n}$. We claim that $E_{2}^{\prime} \subseteq \bigcup_{i \in I}\left\{s_{i} y_{i}, s_{i} z_{i}\right\}$ : Let $e^{\prime} \in E_{2}^{\prime}$. Then its dual is a side of a polygon such that one of its endpoints has an angle that is smaller than $\alpha_{0}$. By the angle analysis above, the only such angles are of $P_{s_{i}}^{\prime}$ at the incenter of $s_{i} y_{i} z_{i}$ for some $i \in I$. So $e^{\prime}$ must be either $s_{i} y_{i}$ or $s_{i} z_{i}$ as needed. Therefore, we can bound:

$$
\sum_{e^{\prime} \in E_{2}^{\prime}} c_{e^{\prime}}^{\prime} \leq \sum_{i \in I}\left(c_{s_{i} y_{i}}^{\prime}+c_{s_{i} z_{i}}^{\prime}\right) \leq \sum_{i \in I} \frac{\varepsilon}{2^{i}} \leq \sum_{i \in \mathbb{N}} \frac{\varepsilon}{2^{i}}=\varepsilon
$$

For the second statement, recall that (for a reference read for example the paragraph preceding Theorem 2.3 of [12]):

$$
\mathrm{C}_{\mathrm{eff}}{ }^{\prime}(\rho \leftrightarrow \infty)=\pi^{\prime}(\rho) \cdot \mathbb{P}_{\rho}^{\prime}[\rho \rightarrow \infty],
$$

where $\pi^{\prime}(\rho)=\sum_{\substack{v \in V^{\prime} \\ v \sim \rho}} c_{\rho v}^{\prime}, \mathbb{P}_{\rho}^{\prime}$ is the probability measure of the weighted random walk on $\left(G^{\prime}, c^{\prime}\right)$ started at $\rho$ and $\rho \rightarrow \infty$ is the event that the random walk never returns to $\rho$. In the circle insertion process we have not added circles in the faces incident to $\rho$ due to the choice of $\alpha_{0}$, and so $\pi(\rho)=\pi^{\prime}(\rho)$. In addition, Corollary 5.9 shows that the weighted random walks on $\left\{C_{v}\right\}_{v \in V^{\prime}}$ started at $\rho$ can be coupled to the weighted random walk on $\left\{C_{v}\right\}_{v \in V}$ by first censoring it to $V$ and then deleting repetitions. In the circle insertion process we have not added vertices adjacent to $\rho$, and so no appearances of $\rho$ will be deleted when censoring and deleting repetitions. Thus, in the coupling we have that the random walker on $G^{\prime}$ returns to $\rho$ iff the random walker on $G$ returns to $\rho$, and so 
$\mathbb{P}_{\rho}[\rho \rightarrow \infty]=\mathbb{P}_{\rho}^{\prime}[\rho \rightarrow \infty]$. We conclude:

$$
\mathrm{C}_{\text {eff }}(\rho \leftrightarrow \infty)=\pi(\rho) \mathbb{P}_{\rho}[\rho \rightarrow \infty]=\pi^{\prime}(\rho) \mathbb{P}_{\rho}^{\prime}[\rho \rightarrow \infty]=\mathrm{C}_{\text {eff }}(\rho \leftrightarrow \infty) .
$$

Finally, the carrier of $\left\{C_{v}\right\}_{v \in V}$ is the same as the carrier of $\left\{C_{v}\right\}_{v \in V}$ since in each face of the original packing we have added finitely many circles.

We are now ready to prove the main result of this paper, restated here:

Theorem 1.3. Let $\Omega \subseteq \mathbb{R}^{2}$ be a parabolic domain. Then for any circle-packed infinite triangulation of $\Omega$, the Dubejko-weighted random walk is recurrent.

Proof. Fix a vertex $\rho \in V$ and let $\varepsilon>0$. We will show that $\mathrm{C}_{\text {eff }}(\rho \leftrightarrow \infty)=0$, which implies recurrence.

Step 1: Insert new circles to the packing: By Lemma 5.16, we may assume without loss of generality that there exists some angle $\alpha_{0}>0$ such that for $E_{2}=\left\{e \in E \mid e\right.$ is not $\alpha_{0}$-nice $\}$ we have $\sum_{e \in E_{2}} c_{e} \leq \varepsilon$ : Indeed, if this is not the case we can replace $\left\{C_{v}\right\}_{v \in V}$ with $\left\{C_{v}^{\prime}\right\}_{v \in V^{\prime}}$ from the Lemma, which has the same carrier and the same effective conductance $\mathrm{C}_{\text {eff }}(\rho \leftrightarrow \infty)$.

Step 2: construct a function $f$ on the vertices: By the parabolicity of $\Omega$, by 5.13 applied to the compact set with nonempty interior $K=P_{\rho}$, there exists a Lipschitz function $\phi: \Omega \rightarrow[0,1]$ compactly supported in $\Omega$ with $\left.\phi\right|_{P_{\rho}} \equiv 1$ such that its Dirichlet energy satisfies:

$$
\iint_{\Omega}\|\nabla \phi\|^{2} d A<\frac{\sin \left(\frac{1}{2} \alpha_{0}\right)}{2 C_{1}\left(\alpha_{0}\right)} \cdot \varepsilon
$$

where $C_{1}\left(\alpha_{0}\right)$ is the constant from Lemma 4.8. By Lemma 5.14, the support of $\phi$ intersects a finite set of polygons $\left\{P_{w}\right\}_{w \in W}$, and denote their union by $A=\bigcup_{w \in W \backslash \rho} P_{w}$. Notice that $\left.\phi\right|_{\partial A} \equiv 0$. Without loss of generality, we may now assume that $\phi$ is harmonic in $\operatorname{int}(A) \backslash P_{\rho}$ : If this is not the case, we can replace the values of $\phi$ there by the unique harmonic solution $\psi$ to the Dirichlet problem with boundary conditions $\left.\psi\right|_{\partial A} \equiv 0$ and $\left.\psi\right|_{\partial P_{\rho}} \equiv 1$. This solution is indeed unique and minimizes the Dirichlet energy due to the piecewise smoothness of the boundary (being a finite union of polygonal lines). Notice that we may still assume that $0 \leq \phi \leq 1$ due to the maximum and minimum principles for harmonic functions. Once $\phi$ is defined, it induces a function $f$ on $V$ by letting $f(v)$ be the value of $\phi$ on the center of $C_{v}$.

Note that under the extra assumption that the continuous function $\phi$ is harmonic, the function $f$ defined here coincides with the function defined in [7] in the proof that parabolicity of the domain implies recurrence of the simple random walk.

Step 3: Bound the discrete energy of $f$ : Recall that $E_{2}=\left\{e \in E \mid e\right.$ is not $\alpha_{0}$-nice $\}$, and set $E_{1}=E \backslash E_{2}$. Then the Dirichlet energy of $f$ is:

$$
\mathcal{E}(f)=\underbrace{\sum_{u v \in E_{1}} c_{u v}(f(v)-f(u))^{2}}_{\mathcal{E}_{1}}+\underbrace{\sum_{u v \in E_{2}} c_{u v}(f(v)-f(u))^{2}}_{\mathcal{E}_{2}} .
$$

By using $0 \leq f \leq 1$ and the choice of $\alpha_{0}$, we can bound the second summand:

$$
\mathcal{E}_{2} \leq \sum_{u v \in E_{2}} c_{u v} \cdot 1 \leq \varepsilon
$$

For any other edge $e=u v \in E_{1}$, denote by $M, N$ the ends of its dual edge, i.e the incenters of the two faces incident to $e$. For any $a, b \in \mathbb{R}^{2}$ denote by $T_{a b}$ the straight line segment connecting $a$ to $b$. For every $z \in T_{M N}$, we can use the identity $(x+y)^{2} \leq 2 x^{2}+2 y^{2}$ to obtain:

$$
c_{u v}(f(v)-f(u))^{2}=c_{u v}(\phi(v)-\phi(u))^{2} \leq 2 c_{u v}(\phi(v)-\phi(z))^{2}+2 c_{u v}(\phi(u)-\phi(z))^{2} .
$$


Since this inequality holds for any $z \in T_{M N}$, it also holds in expectation:

$$
c_{u v}(f(v)-f(u))^{2} \leq \frac{2 c_{u v}}{\left|T_{M N}\right|} \int_{T_{M N}}(\phi(v)-\phi(z))^{2} d z+\frac{2 c_{u v}}{\left|T_{M N}\right|} \int_{T_{M N}}(\phi(u)-\phi(z))^{2} d z .
$$

We continue to bound the first summand above. Since $\phi$ is smooth except maybe at the boundaries of polygons, we can write $\phi(v)-\phi(z)=\int_{T_{v z}} \nabla \phi(\underline{r}) d \underline{r}$. Thus, using the definition of $c_{u v}$ and Cauchy-Schwarz for line integrals we bound:

$$
\frac{2 c_{u v}}{\left|T_{M N}\right|} \int_{T_{M N}}(\phi(v)-\phi(z))^{2} d z=\frac{2}{\left|T_{u v}\right|} \int_{T_{M N}}\left(\int_{T_{v z}} \nabla \phi(\underline{r}) d \underline{r}\right)^{2} d z \leq 2 \int_{T_{M N}} \frac{\left|T_{v z}\right|}{\left|T_{u v}\right|} \int_{T_{v z}}\|\nabla \phi(\underline{r})\|^{2} d r d z .
$$

Since the angles $\measuredangle v z M$ and $\measuredangle v z N$ add up to $\pi$ radians, one of these angles is at least $\frac{\pi}{2}$. Using this and $\left|T_{u v}\right| \geq r_{v}$ we bound:

$$
\frac{\left|T_{v z}\right|}{\left|T_{u v}\right|} \leq \max \left\{\frac{\left|T_{v M}\right|}{r_{v}}, \frac{\left|T_{v N}\right|}{r_{v}}\right\}=\max \left\{\frac{1}{\sin \measuredangle v M N}, \frac{1}{\sin \measuredangle v N M}\right\} \leq \frac{1}{\sin \left(\frac{1}{2} \alpha_{0}\right)},
$$

where the last inequality holds because $u v \in E_{1}$ and hence $T_{M N}$ is $\alpha_{0}$-nice for both $P_{u}$ and $P_{v}$. Using this and a similar calculation for $u$ instead of $v$, we get:

$$
c_{u v}(f(v)-f(u))^{2} \leq \frac{2}{\sin \left(\frac{1}{2} \alpha_{0}\right)}\left(\int_{T_{M N}} \int_{T_{v z}}\|\nabla \phi(\underline{r})\|^{2} d r d z+\iint_{T_{M N}}\|\nabla \phi(\underline{r})\|^{2} d r d z\right) .
$$

For each $v \in V$ set $X_{v}$ to be the set of sides of $P_{v}$ that are dual to some edge $v u \in E_{1}$. Then summing up the last inequality over all $u v \in E_{1}$ and using Lemma 4.10 we bound:

$$
\begin{aligned}
\mathcal{E}_{1} & \leq \frac{2}{\sin \left(\frac{1}{2} \alpha_{0}\right)} \cdot \sum_{v \in V} \sum_{S \in X_{v}}\left(\int_{S} \int_{T_{v z}}\|\nabla \phi(\underline{r})\|^{2} d r d z\right) \leq \frac{2}{\sin \left(\frac{1}{2} \alpha_{0}\right)} \cdot \sum_{v \in V} C_{1}\left(\alpha_{0}\right) \iint_{P_{v}}\|\nabla \phi\|^{2} d A \leq \\
& \leq \frac{2 C_{1}\left(\alpha_{0}\right)}{\sin \left(\frac{1}{2} \alpha_{0}\right)} \cdot \iint_{\Omega}\|\nabla \phi\|^{2} d A \leq \varepsilon .
\end{aligned}
$$

Combining inequalities 5.3 and 5.4 we can finally bound the Dirichlet energy of $f$ :

$$
\mathcal{E}(f)=\mathcal{E}_{1}+\mathcal{E}_{2} \leq 2 \varepsilon .
$$

Step 5: Deducing recurrence: We've found a function $f: V \rightarrow \mathbb{R}$ with $\mathcal{E}(f) \leq 2 \varepsilon$. Furthermore, since $\phi$ vanishes outside a finite union of polygons, $f$ is finitely supported. Lastly, $f(\rho)=1$. By Dirichlet's Principle, the effective conductance in the network $(G, c)$ satisfies $\mathrm{C}_{\text {eff }}(\rho \leftrightarrow \infty) \leq 2 \varepsilon$. Since $\varepsilon$ is arbitrary, $\mathrm{C}_{\text {eff }}(\rho \leftrightarrow \infty)=0$ and hence the network is recurrent.

\section{Extension to Higher Dimensions}

The goal of this section is to define a higher-dimensional analogue of the Dubejko weights and prove Proposition 6.4, showing that the weighted random walk is a martingale.

Definition 6.1. Given a set of spheres $\left\{S_{v}\right\}_{v \in V}$ in $\mathbb{R}^{3}$ with disjoint interiors, we can define its tangency graph: The vertex set is $V$ and two vertices $v, u \in V$ are connected by an edge iff their spheres $S_{v}$ and $S_{u}$ are tangent. Given two neighbouring vertices $u, v \in V$, we define their common tangent plane to be the unique plane in $\mathbb{R}^{3}$ that is tangent to both $S_{v}$ and $S_{u}$. For each neighbour of some $v \in V$, their common tangent plane cuts $\mathbb{R}^{3}$ into two half-spaces, one of them containing $v$. If the intersection over all the neighbours of $v$ of the half-spaces containing $v$ is bounded, we say that $v$ is covered and call the intersection the polyhedron of $v$ denoted by $P_{v}$. We say that $\left\{S_{v}\right\}_{v \in V}$ is covering if all vertices are covered and for every edge $u v \in E$ their respective polyhedra intersect along a common face. 
Definition 6.2. Let $\left\{S_{v}\right\}_{v \in V}$ be a covering 3-sphere packing with tangency graph $G=(V, E)$. Then in a similar fashion to the Dubejko weights, we define the weight of an edge $u v \in E$ to be:

$$
c_{u v}:=\frac{A_{u v}}{\|v-u\|}
$$

where $A_{u v}$ is the area of the shared face of $P_{u}$ and $P_{v}$.

Remark 6.3. Similar definitions can be made in dimensions $d>3$, where instead of the area we use the $d-1$ dimensional volume of the shared face of the polytopes of $P_{u}$ and $P_{v}$, and the following Proposition would remain true.

Proposition 6.4. Let $\left\{S_{v}\right\}_{v \in V}$ be a covering 3-sphere packing with tangency graph $G=(V, E)$. Identify the vertices of $V$ with the centers of the their spheres, and let $\left(Z_{n}\right)_{n \in \mathbb{N}}$ be the sequence of centers of spheres visited in a random walk weighted according to Equation 6.1 started at some vertex $v_{0} \in V$. Then $\left(Z_{n}\right)_{n \in \mathbb{N}}$ is a martingale.

Proof. Set $\pi(x)=\sum_{y \sim x} c_{x y}$ and denote the transition probabilities by $P_{x, y}=\frac{c_{x y}}{\pi(x)}$. Let $v \in V$, and let $u_{1}, u_{2}, \ldots, u_{n} \in V$ be its neighbors in $G$. It is enough to show:

$$
\sum_{i=1}^{n} P_{v, u_{i}} \cdot\left(u_{i}-v\right)=0 .
$$

Plugging in the definition of the weights, we get:

$$
\sum_{i=1}^{n} P_{v, u_{i}} \cdot\left(u_{i}-v\right)=\frac{1}{\pi(v)} \sum_{i=1}^{n} A_{u_{i} v} \frac{u_{i}-v}{\left\|u_{i}-v\right\|} .
$$

Write $\partial P_{v}=\bigcup_{i=1}^{n} Q_{v u_{i}}$, where $Q_{v u_{i}}$ is the common face of $P_{v}$ and $P_{u_{i}}$, and notice that $\frac{u_{i}-v}{\left\|u_{i}-v\right\|}$ is the outwardpointing normal of $Q_{v u_{i}}$. Thus, we can write:

$$
\sum_{i=1}^{n} A_{u_{i} v} \frac{u_{i}-v}{\left\|u_{i}-v\right\|}=\sum_{i=1}^{n} \operatorname{Area}\left(Q_{v u_{i}}\right) \cdot \hat{n}_{Q_{v u_{i}}} .
$$

We wish to show that this vector is zero. Let $\hat{m}$ be any unit vector, then using the divergence theorem and the fact that the divergence of a constant vector field is zero we find:

$$
\hat{m} \cdot\left(\sum_{i=1}^{n} \operatorname{Area}\left(Q_{v u_{i}}\right) \cdot \hat{n}_{Q_{v u_{i}}}\right)=\oiint_{\partial P_{v}} \hat{m} \cdot d S=\iiint_{P_{v}}(\nabla \cdot \hat{m}) d V=0 .
$$

Since this is true for any unit vector $\hat{m}$, we have $\sum_{i=1}^{n} A_{u_{i} v} \frac{u_{i}-v}{\left\|u_{i}-v\right\|}=0$ as needed.

Remark 6.5. The second part of the last proof is simply the fact that the vector area of any closed surface is zero. A physical interpretation of this claim is the following: Fill a metallic shell in the shape of $\partial P_{v}$ with water and put it somewhere far away in outer space. The force acting on each face due to the water pressure is proportional to its area and acts outwards. Hence, $\sum_{i=1}^{n} \operatorname{Area}\left(Q_{v u_{i}}\right) \cdot \hat{n}_{Q_{v u_{i}}}=0$ is exactly the claim that the net force acting on the shell is zero - or in other words that the shell would not start accelerating spontaneously in some direction.

Acknowledgements. The authors wish to thank Asaf Nachmias for many useful discussions and comments.

\section{REFERENCES}

[1] Sheldon Axler, Paul Bourdon, and Ramey Wade. Harmonic function theory, volume 137. Springer Science \& Business Media, 2013.

[2] Harold Scott Macdonald Coxeter, Harold Scott Macdonald Coxeter, Harold Scott Macdonald Coxeter, and Harold Scott Macdonald Coxeter. Introduction to geometry, volume 136. Wiley New York, 1969.

[3] Tomasz Dubejko. Random walks on circle packings. Contemporary Mathematics, 211:169-182, 1997.

[4] Richard James Duffin. Potential theory on a rhombic lattice. Journal of Combinatorial Theory, 5(3):258-272, 1968.

[5] Rick Durrett. Probability: theory and examples. Cambridge university press, 2010. 
[6] Alexander Grigor'yan. Analytic and geometric background of recurrence and non-explosion of the brownian motion on riemannian manifolds. Bulletin of the American Mathematical Society, 36(2):135-249, 1999.

[7] Ori Gurel-Gurevich, Asaf Nachmias, and Juan Souto. Recurrence of multiply-ended planar triangulations. Electronic Communications in Probability, 22, 2017.

[8] Zheng-Xu He and Oded Schramm. Fixed points, koebe uniformization and circle packings. Annals of Mathematics, 137(2):369-406, 1993.

[9] Zheng-Xu He and Oded Schramm. Hyperbolic and parabolic packings. Discrete \& Computational Geometry, 14(2):123-149, 1995.

[10] John G Kemeny, J Laurie Snell, and Anthony W Knapp. Denumerable Markov chains: with a chapter of Markov random fields by David Griffeath, volume 40. Springer Science \& Business Media, 2012.

[11] Paul Koebe. Kontaktprobleme der konformen Abbildung. Hirzel, 1936.

[12] Russell Lyons and Yuval Peres. Probability on trees and networks, volume 42. Cambridge University Press, 2017.

[13] Asaf Nachmias. Planar maps, random walks and circle packing. arXiv preprint arXiv:1812.11224, 2018.

[14] Burt Rodin and Dennis Sullivan. The convergence of circle packings to the riemann mapping. Journal of Differential Geometry, 26(2):349-360, 1987.

[15] Kenneth Stephenson. Introduction to circle packing: The theory of discrete analytic functions. Cambridge University Press, 2005.

[16] Wolfgang Woess. Random walks on infinite graphs and groups, volume 138. Cambridge university press, 2000. 